\section{Pacific Northwest}

National Laboratory

Operated by Battelle for the

U.S. Department of Energy

\title{
Remote Chemical Sensing Using Quantum Cascade Lasers
}

\author{
W. W. Harper \\ P. M. Aker \\ J. D. Strasburg \\ J. F. Schultz
}

January 2004

Prepared for the U.S. Department of Energy

under Contract DE-AC06-76RL01830 


\title{
DISCLAIMER
}

This report was prepared as an account of work sponsored by an agency of the United States Government. Neither the United States Government nor any agency thereof, nor Battelle Memorial Institute, nor any of their employees, makes any warranty, express or implied, or assumes any legal liability or responsibility for the accuracy, completeness, or usefulness of any information, apparatus, product, or process disclosed, or represents that its use would not infringe privately owned rights. Reference herein to any specific commercial product, process, or service by trade name, trademark, manufacturer, or otherwise does not necessarily constitute or imply its endorsement, recommendation, or favoring by the United States Government or any agency thereof, or Battelle Memorial Institute. The views and opinions of authors expressed herein do not necessarily state or reflect those of the United States Government or any agency thereof.

\author{
PACIFIC NORTHWEST NATIONAL LABORATORY \\ operated by \\ BATTELLE \\ for the \\ UNITED STATES DEPARTMENT OF ENERGY \\ under Contract DE-AC06-76RL01830
}

Printed in the United States of America

$$
\begin{gathered}
\text { Available to DOE and DOE contractors from the } \\
\text { Office of Scientific and Technical Information, } \\
\text { P.O. Box 62, Oak Ridge, TN 37831-0062; } \\
\text { ph: (865) 576-8401 } \\
\text { fax: }(865) \text { 576-5728 } \\
\text { email: reports@adonis.osti.gov }
\end{gathered}
$$

\author{
Available to the public from the National Technical Information Service, \\ U.S. Department of Commerce, 5285 Port Royal Rd., Springfield, VA 22161 \\ ph: (800) 553-6847 \\ fax: $(703) 605-6900$ \\ email: orders@ntis.fedworld.gov \\ online ordering: http://www.ntis.gov/ordering.htm
}

This document was printed on recycled paper. 


\title{
Remote Chemical Sensing Using Quantum Cascade Lasers
}

\author{
W. W. Harper \\ J. D. Strasburg \\ P. M. Aker \\ J. F. Schultz
}

January 2004

Prepared for

the U.S. Department of Energy

under Contract DE-AC06-76RL01830

Pacific Northwest National Laboratory

Richland, Washington 99352 


\section{Summary}

Research done by the IR sensors team at PNNL is focused on developing advanced spectroscopic methods for detecting signatures of nuclear, chemical, biological and explosives weapons or weapons production. The sensors we develop fall into two categories: remote sensors that can be operated at distances ranging from $150 \mathrm{~m}$ to $10 \mathrm{~km}$, and point sensors that are used for in-situ inspection and detection.

Theoretical modeling studies done at PNNL in FY00 identified frequency-modulated differential absorption LIDAR (FM DIAL) as a promising candidate for remote sensing applications. Laboratory experiments conducted in FY01 and preliminary field tests, conducted with a prototype portable system in FY02, confirmed the theoretical conclusions. FY03 has seen an explosion in FM DIAL progress with the net result being solid confirmation that FM DIAL is a technique capable of remote chemical monitoring in a wide variety of venues. For example, FM DIAL was used to detect a small plume of hydrogen sulfide, a candidate $\mathrm{CW}$ agent, released in the desert environment of the Hanford 200 Area site. These experiments were conducted over a range of physical conditions including outside temperatures ranging from $70^{\circ} \mathrm{F}$ to $105^{\circ} \mathrm{F}$ and turbulence conditions ranging from quiescent to chaotic. We are now rapidly developing the information needed to design prototype FM DIAL systems that are optimized for specific applications that include scenarios such as fixed position stand-off detection and mobile UAV mounted remote monitoring. Just as an example, in FY04 we will use FM DIAL to detect both in-facility and outdoor release of enriched $\mathrm{UF}_{6}$.

The rapid progress in FM DIAL research made in FY03 is attributed to several advances. First, final construction of a custom-designed trailer allowed the instrument to be housed in a mobile temperaturecontrolled environment. This allowed the experiment to be transported to several locations so that data could be collected under a range of physical conditions, which has led to a better understanding of a variety of experimental noise sources. With this knowledge, we have been able to implement several changes in the way the FM DIAL data is collected and processed, with the net result being a drastic improvement in our confidence of analyte concentration measurement and an improvement in the instrument detection limit.

The range of chemicals detectable by FM DIAL has also been extended. Prior to FY03 only water and nitrous oxide $\left(\mathrm{N}_{2} \mathrm{O}\right)$ had been seen. Experiments on extending the tuning range of the quantum cascade laser (QCL) currently used in the experiments demonstrate that many more species are now accessible including $\mathrm{H}_{2} \mathrm{~S}, \mathrm{C}_{2} \mathrm{~F}_{4} \mathrm{H}_{2}$, and $\mathrm{CH}_{4}$. We additionally demonstrated that FM DIAL measurements can be made using short wave infrared (SWIR) telecommunications lasers. While measurements made using these components are noisier because turbulence and particulate matter cause more interference in this spectral region, monitoring in this region enables larger species to be detected simply because these lasers have a greater tuning range. In addition, SWIR monitoring also allows for the detection of secondrow hydride species such as $\mathrm{HF}$ and $\mathrm{HCl}$, which are important nuclear and $\mathrm{CWA}$ proliferation signatures. 


\section{Contents}

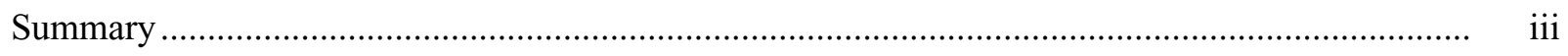

1.0 Frequency Modulated Differential Absorption Lidar (FM DIAL) Remote Sensor.................. 1.1

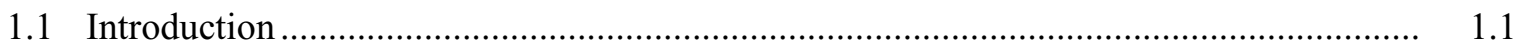

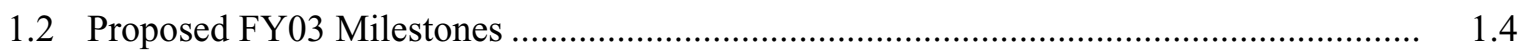

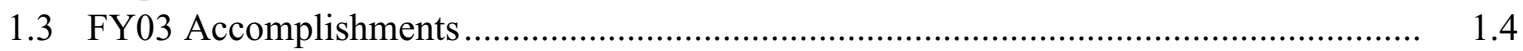

2.0 Final Incorporation of the FM DIAL Experiment in a Mobile Trailer ................................. 2.1

2.1 Optical-Mechanical Design ................................................................................ 2.1

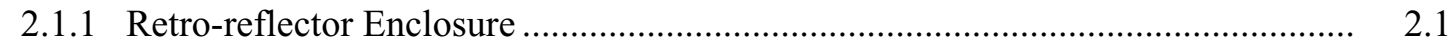

2.1.2 Acme-screw Jacking System........................................................................ 2.1

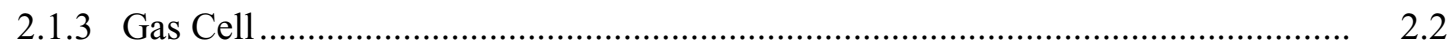

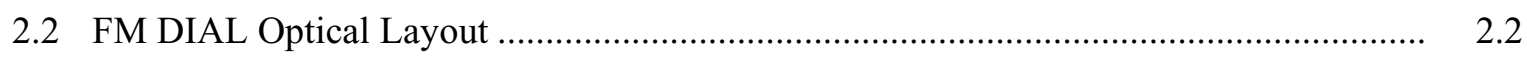

3.0 Enhancements in FM DIAL Versatility ..................................................................

3.1 Dual QCL FM DIAL Experiments....................................................................... 3.1

3.2 Addition of SWIR Detection Capability ................................................................... 3.2

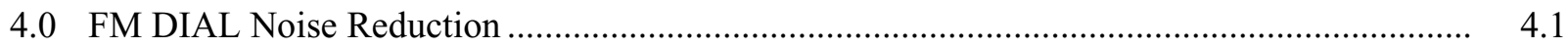

4.1 Understanding Error Propagation in FM DIAL ........................................................

4.2 Single Beam Noise Subtraction and Intensity Normalization ........................................ 4.3

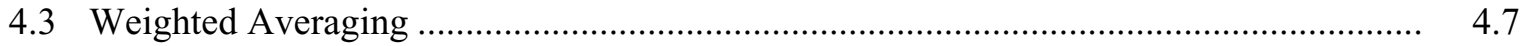

4.4 Influence of Turbulence on FM DIAL Signal to Noise ................................................ 4.13

4.4.1 Dependence of Range and Sweep Rate on FM DIAL Performance ..................... 4.13

4.4.2 Effects of Atmospheric Turbulence …............................................................... 4.17

4.4.3 Quantifying Atmospheric Turbulence .............................................................. 4.19

4.5 QCL Beam Spread and Relation to Experimental Noise .............................................. 4.22

4.6 Impact of Large Objects Moving Through the QCL Beam ......................................... 4.24

5.0 Real Time Data Acquisition and Analysis.............................................................. 5.1

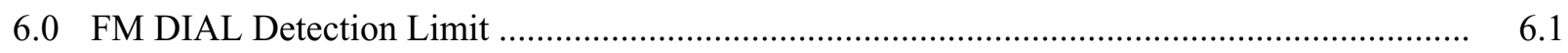

$7.0 \quad$ Chemical Release Experiments ............................................................................. 7.1 


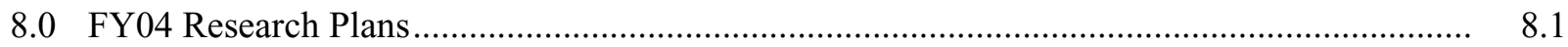

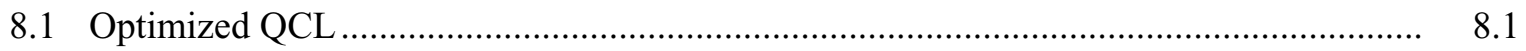

8.2 FM DIAL Performance and Atmospheric Turbulence.................................................. 8.1

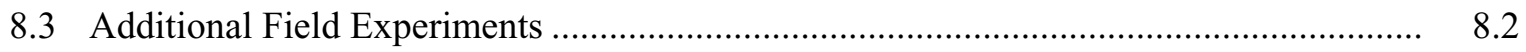

8.4 Pulsed Broadband FM DIAL Feasibility ................................................................ 8.2

8.5 FM DIAL Collection Geometry ........................................................................... 8.2 


\section{Figures}

1.1 The Linear Absorption Curve is Given by the Black Line, the First Frequency

Modulated Derivative is Given by the Red Line

1.2 The Solid Black Line is the Average of 512 FM DIAL Traces Taken

When $\mathrm{N}_{2} \mathrm{O}$ Gas was Released

2.1 Retro-Reflector Array Mounted in the NEMA 4X Enclosure

2.2 One of Four Tripod Jacks Used to Isolate the Optical Table.

2.3 Brewster Angle Gas Cell

2.4 FM DIAL Optical Layout.

3.1 Data that was Collected from Two QC Lasers Simultaneously.

4.1 Plots of the FM and RAM Intensity Data, the Ratio $f$, and the Uncertainty $\Delta f$ for the Three Cases Just Outlined in the Text

4.2 Single Beam Experimental Setup for Lock-In Detection

4.4 FM and RAM Lock-In Signals Observed When a Mechanical Chopper is Placed in the Laser Beam Path.

4.5 Ratio of the FM and RAM Traces Shown in Figure 4.4

4.6 Influence of Atmospheric Turbulence on FM DIAL Returned Intensity

4.7 Data Set Used in Averaging Technique Comparison

4.8 Generated Standard Deviations that Range from 0.0025 to 0.5 .

4.9 Results from Averaging 10 Different Traces

4.11 Top Panel Shows Raw Data from the $4.67 \mathrm{~km}$ Retro-Reflector, Consisting of Two Water Derivative Absorption Features. 
4.12 Top and Bottom Panels Show the Peak Shape Height and the Estimated Uncertainty for Each Measurement, Respectively

4.13 Signal to Noise for the $4.67 \mathrm{~km}$ Retro are Indicated by the Circles

4.14 Effect of Atmospheric Turbulence on Laser Beam Footprint.

4.15 Raw Atmospheric Turbulence Data for July 18, 2003 at 6:30 AM and 1:05 PM.

4.16 Autocorrelation Data for July 18, 2003 at 6:30 AM and 1:05 PM

4.17 Scintillation Index from 5 AM to 2 PM on Three Different Days in July 2003

4.18 Time Scale When Subsequent Points are 50\% Correlated from 5 AM to 2 PM from Three Different Days in July

4.19 Measurement of the Focal Spot Width and Comparison with

Diffraction Limited Airy Pattern

4.20 Angular Beam Profiles Taken from a Retro-Reflector Positioned at $2.4 \mathrm{~km}$ and $5.4 \mathrm{~km}$

4.21 One of the Traces in a Sequence of Data Showing Reduced Intensity Returns at $0.4 \mathrm{~s}$ Due to Partial Laser Beam Blockage.

4.22 Resulting Trace After Normalizing the Data in Figure 4.21, FM Divided by RAM

4.23 Geometry of Object Passing Through a Beam Path

5.1 Autocorrelation Calculation.

5.2 Average Calculation

7.1 View from the Retro-Reflector Toward the Trailer.

$7.2 \quad \mathrm{H}_{2} \mathrm{~S}$ Release Skid

7.3 FM DIAL Trailer and the FTIR Sensor System .....

7.4 Selected Averaged Waveforms Showing the Derivative Absorption Features Due to $\mathrm{H}_{2} \mathrm{~S}$......

7.5 Results from the Extended Lease Squares Treatment. 


\subsection{Frequency Modulated Differential Absorption Lidar (FM DIAL) Remote Sensor}

\subsection{Introduction}

Differential absorption lidar (DIAL) is a remote sensing technique which uses two lasers, one having a wavelength on and the other having a wavelength off, a target molecular species absorption line. Chemical absorbance (and by extension concentration) measurements are obtained by directing the two wavelengths into the atmosphere, scattering the light off a remote target, and measuring differences in the returned optical energies. Frequency modulated (FM) DIAL is somewhat different in that it uses only one laser, specifically one that is wavelength tunable, but similar in that differential absorption information is obtained by sweeping the laser wavelength, or frequency as is often used in the spectroscopy community, on and off an absorption line. PNNL uses quantum cascade lasers (QCLs) in their FM DIAL experiment, but other lasers, such as diode lasers and lead-salt lasers can also be incorporated. QCL and diode lasers can be frequency tuned by adjusting the amount of bias current supplied to the device.

The concept of frequency modulated spectroscopy, and more specifically FM DIAL, is pictured in Figure 1.1. To generate an FM DIAL spectrum, the center frequency of a probe laser is slowly scanned across a molecular absorption feature, which is given by the lower curve in Figure 1.1a. While the central frequency is being scanned, an additional frequency modulation, $\Delta \omega$, at much higher repetition rate, is imposed on the probe beam. This second frequency modulation, as illustrated in Figure 1.1b, shows up as an amplitude modulation in the returning beam. Using a lock-in detection scheme, the amplitude modulation, $\Delta I$, is monitored as a function of the incident laser optical frequency to generate the first FM derivative curve that is shown by the solid red line in Figure 1.1a.

The basic idea behind the FM DIAL experiments PNNL performs consists of directing a laser toward a scattering target, collecting the scattered photons using a telescope, and analyzing the data to estimate a targeted analyte's concentration over the pathlength the laser light traversed. This is similar to having a laser traverse a long pathlength absorption cell and focus onto a detector. In the latter experiment the analyte concentration is determined using the relation between absorbance and concentration, and Beer's law,

$$
n \alpha L=A=-\ln \left(\frac{I-\Delta I}{I}\right)
$$

Here $n$ is number density in molecules $\mathrm{cm}^{-3}, \alpha$ is the absorption cross section in $\mathrm{cm}^{2}$ molecule ${ }^{-1}, L$ is the pathlength in $\mathrm{cm}, I$ is the incident power, and $\Delta I$ is the absorbed power. Often $I-\Delta I$ is measured directly as the intensity that is transmitted through the sample. The smallest absorbance that can be measured is related to the smallest $\Delta I$ that can be measured. For an estimate of the absorbance, $I$ must be measured, along with either $(I-\Delta I)$ or simply $\Delta I$. Most simple techniques measure $I$ and $I-\Delta I$ directly, but for small 


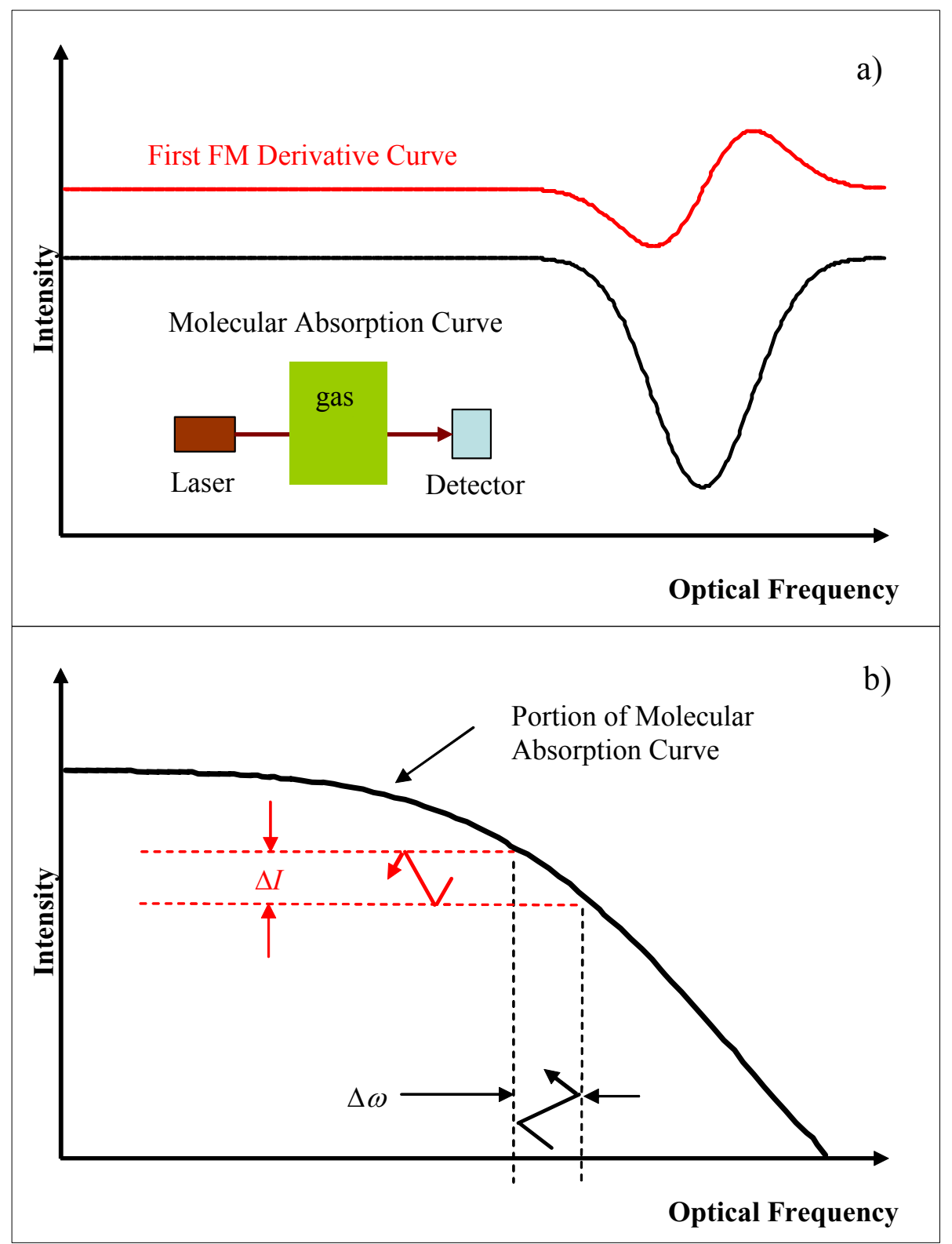

Figure 1.1. a) The Linear Absorption Curve is Given by the Black Line, the First Frequency Modulated Derivative is Given by the Red Line. The upper curve has been shifted upward for clarification purposes. b) Pictorial depiction of how frequency (or wavelength) modulating the incident light produces an amplitude modulation in the returning light of an FM DIAL type of experiment.

$\Delta I$ it is exceedingly difficult to distinguish a difference between the two quantities. Frequency modulation (FM) techniques are very sensitive to measuring $\Delta I$ directly, but do not provide a direct measurement of $I$. 
In late FY02 we proposed a way to estimate the absorbance observed in an FM DIAL experiment. The absorbance estimator equation uses the residual amplitude modulation (RAM) that results when QCLs or diode lasers are frequency modulated. RAM is usually regarded as a nuisance for sensors; however, in our case it proves to be extremely useful as it eliminates the need to do double modulation experiments. The problem is that the calculation of absorbance requires the measurement of $\Delta I$ as well as I. Using FM, a derivative technique, the laser dc return signal is not measured directly. However, the QCL has a nearly linear power curve (the slope efficiency), and the dc laser return is proportional to the observed RAM signal. Figure 1.2 shows the result of data collected when nitrous oxide gas was released into the atmosphere. The $\mathrm{x}$-axis in Figure 1.2 is given in time units as the laser current and wavelength are ramped. The laser wavelength is a slightly nonlinear function of laser drive current, and the current is a linear function of time. In all cases, the laser is ramped over approximately $1.5 \mathrm{~cm}^{-1}$ (current ranges from $0.2 \mathrm{~A}$ to $0.8 \mathrm{~A}$ ), with increasing wavenumber to the left (shorter time). Subsequent figures of FMDIAL data are plotted as a function of time in the same way as Figure 1.2.

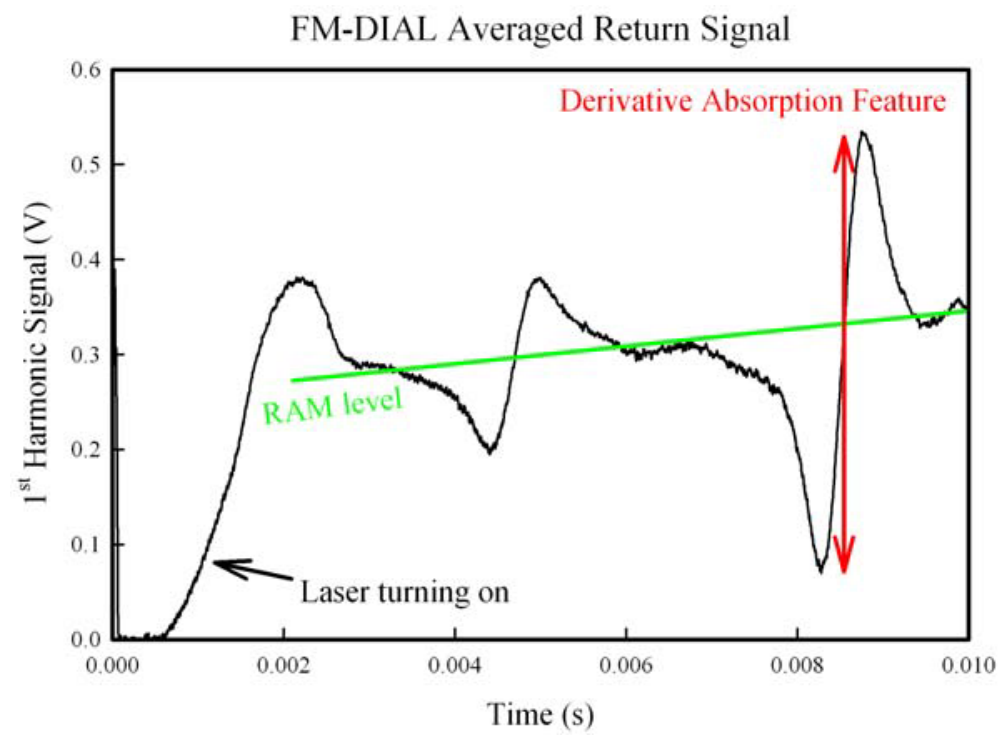

Figure 1.2. The Solid Black Line is the Average of 512 FM DIAL Traces Taken When $\mathrm{N}_{2} \mathrm{O}$ Gas was Released. Initially the laser is off, it is then frequency tuned (in time) over two $\mathrm{N}_{2} \mathrm{O}$ absorptions that show up as derivative line shapes at about 0.0045 and $0.0085 \mathrm{sec}$. The RAM level is shown as a green line, and the magnitude of the derivative signal from the absorption feature is shown in red.

The data from Figure 1.2 may be used to estimate the observed absorbance, $A$, according to the equation:

$$
A=\frac{I_{m}\left(V_{1 \max }-V_{1 \min }\right)}{2 h_{1} V_{o f f}\left(I_{0}-I_{T}\right)},
$$


where $h_{1}$ is a measurable constant that is unique to a particular FM pattern, $I_{m}$ is the laser current modulation, $I_{0}$ is laser current at the peak position, $I_{T}$ is the laser threshold current, $V_{1 \max }$ is the maximum of the first derivative signal, $V_{1 \min }$ is the minimum of the first derivative signal, and $V_{\text {off }}$ is the RAM level at the peak center. This equation has been simplified using the small absorbance approximation; however, a more general equation may be used as well.

\subsection{Proposed FY03 Milestones}

As outlined in our FY02 final year report our overarching goal for this year's research was to advance the FM DIAL experiment to the point where a comparison could be made between observed and theoretically modeled instrument performance, and to use and understand any differences noted to improve the instrument's response and sensitivity. Key to achieving this objective was passing milestones that included:

1. Housing the instrument in an environmentally controlled trailer,

2. Improving FM DIAL versatility by improving QCL tuning range and/or adding additional wavelengths to the experiment,

3. Testing the effect that a number of noise reduction techniques associated with data collection and analysis have on concentration estimate confidence,

4. Incorporating a computer-controlled data acquisition and real-time concentration analysis system in the experiment,

5. Determining detection limits for a bistatic (retroreflector) configuration,

6. Testing the efficacy of FM DIAL for chemical warfare agent (CWA) attack warning.

We met all of these milestones, and the results of our efforts demonstrate that FM DIAL spectroscopy is an extremely effective technique for performing remote sensing of NBC weapons and explosives proliferation. The next section of this report outlines our accomplishments in detail.

\subsection{FY03 Accomplishments}

FY03 has seen much advancement to the FM DIAL program. Accomplishments include completion of the mobile trailer, incorporation of multiple lasers into the system, improvement in noise reduction techniques, implementation of real-time data acquisition and analysis software, further examination of the achievable detection limits and performance of several field tests including chemical release experiments. Each of these accomplishments will be described in detail in the following sections. 


\subsection{Final Incorporation of the FM DIAL Experiment in a Mobile Trailer}

\subsection{Optical-Mechanical Design}

While most of the trailer design and construction was completed in FY02, a few small projects had to be completed to optimize the trailer for remote sensing experiments. These included constructing a retroreflector array enclosure, custom designing and building jacks to stabilize the FM DIAL optical bench, and designing and fabricating a high vacuum cell that can be used for FM DIAL laser calibration and absolute sensitivity measurements.

\subsubsection{Retro-reflector Enclosure}

In remote sensing applications, a retro-reflector is often placed in the path of the laser beam to boost the return signal intensity. A retriever telescope is aligned with the retro-reflector to collect as much of the scattered light as possible. A large retro-reflector array, shown in Figure 2.1, was procured and installed inside a NEMA 4X enclosure to protect it from dust and rain and allow the array to be attached to a large tripod for field use.

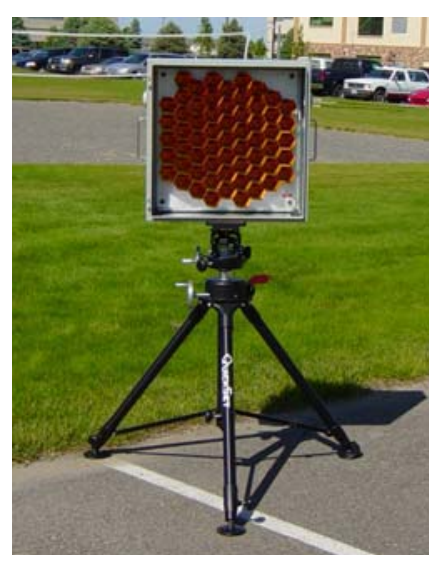

Figure 2.1. Retro-Reflector Array Mounted in the NEMA 4X Enclosure (door removed)

\subsubsection{Acme-screw Jacking System}

A custom jacking system was designed for the FM DIAL optical bench which is housed in the trailer. The jacking system fixes the optical table directly to the ground through holes in the trailer floor. The jacking system allows infrared detection experiments to be conducted at very long range (up to $10 \mathrm{~km}$ ) because it eliminates mechanical jitter caused by wind or personnel movement within the trailer. The jack, pictured in Figure 2.2, was fabricated at a reasonable cost by incorporating precision acme screws into a commercially available tripod jack. 


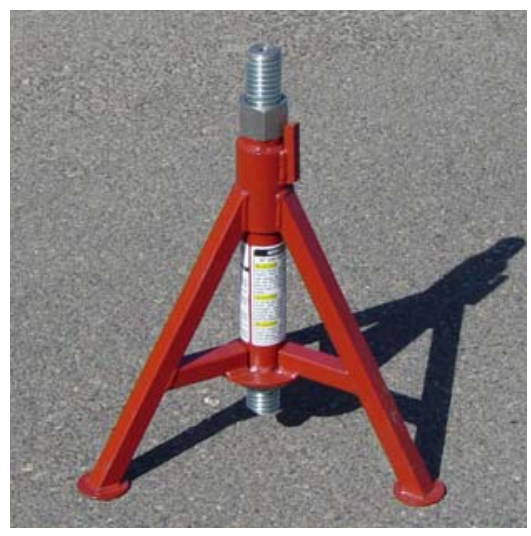

Figure 2.2. One of Four Tripod Jacks Used to Isolate the Optical Table

\subsubsection{Gas Cell}

A gas cell with optical windows at Brewster's angle was also completed in FY03, to minimize optical fringing. The cell, pictured in Figure 2.3, can contain about 1 liter of gas and will serve as a target for chemical detection. Barium fluoride windows are installed at each end of the cylinder enabling low loss IR transmission throughout the 3.5-12 $\mu \mathrm{m}$ range. The windows are oriented at $55^{\circ}$ (Brewster's Angle) to the longitudinal axis of the cylinder to maximize transmission and minimize back reflection. The windows are also wedged at 30 arc minutes to further reduce fringing interference effects. The cylinder will normally be used at atmospheric pressure, but can be used in high vacuum applications as well.

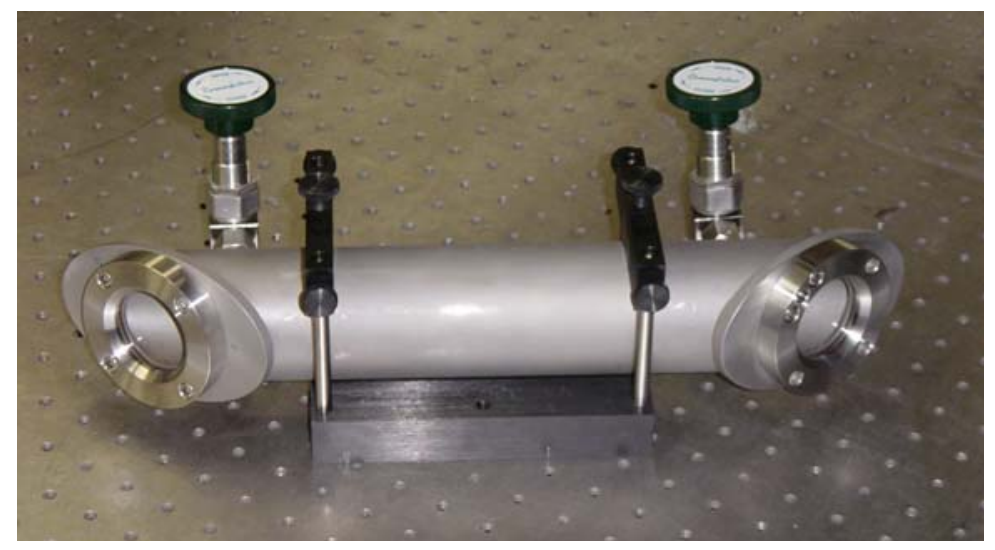

Figure 2.3. Brewster Angle Gas Cell

\subsection{FM DIAL Optical Layout}

Figure 2.4 depicts the experimental layout of the FM DIAL experiment. The main laser source is a long wave infrared (LWIR), $8.2 \mu \mathrm{m}$ quantum cascade (QC) laser that is capable of outputting $100 \mathrm{~mW}$ of $\mathrm{cw}$ power. The laser output is adjusted to have a $1 \mathrm{mrad}$ (diffraction limited) divergence. A portion of the beam is split off to go to diagnostics that include a Germanium reference etalon and various gas 
reference cells. The Germanium etalon has a free spectral range of $0.0475 \mathrm{~cm}^{-1}$ and a finesse of approximately 3 . This provides about 20 reference fringe markers to monitor the laser wavelength. Different reference gas cells have been used in different experiments. Often it is useful to have a lowpressure nitrous oxide cell for determining the absolute operating wavelength. It is also useful to have a sample of the chemical to be detected at atmospheric pressure to simulate actual signal returns. The receiver telescope is a Newtonian reflector with a $10^{\prime \prime} \mathrm{f}-3$ primary mirror. The primary MCT detector is a $1 \mathrm{~mm}$ circular detector element. The large mirror can be adjusted to direct the output beam and telescope field-of-view using a gimble mount.

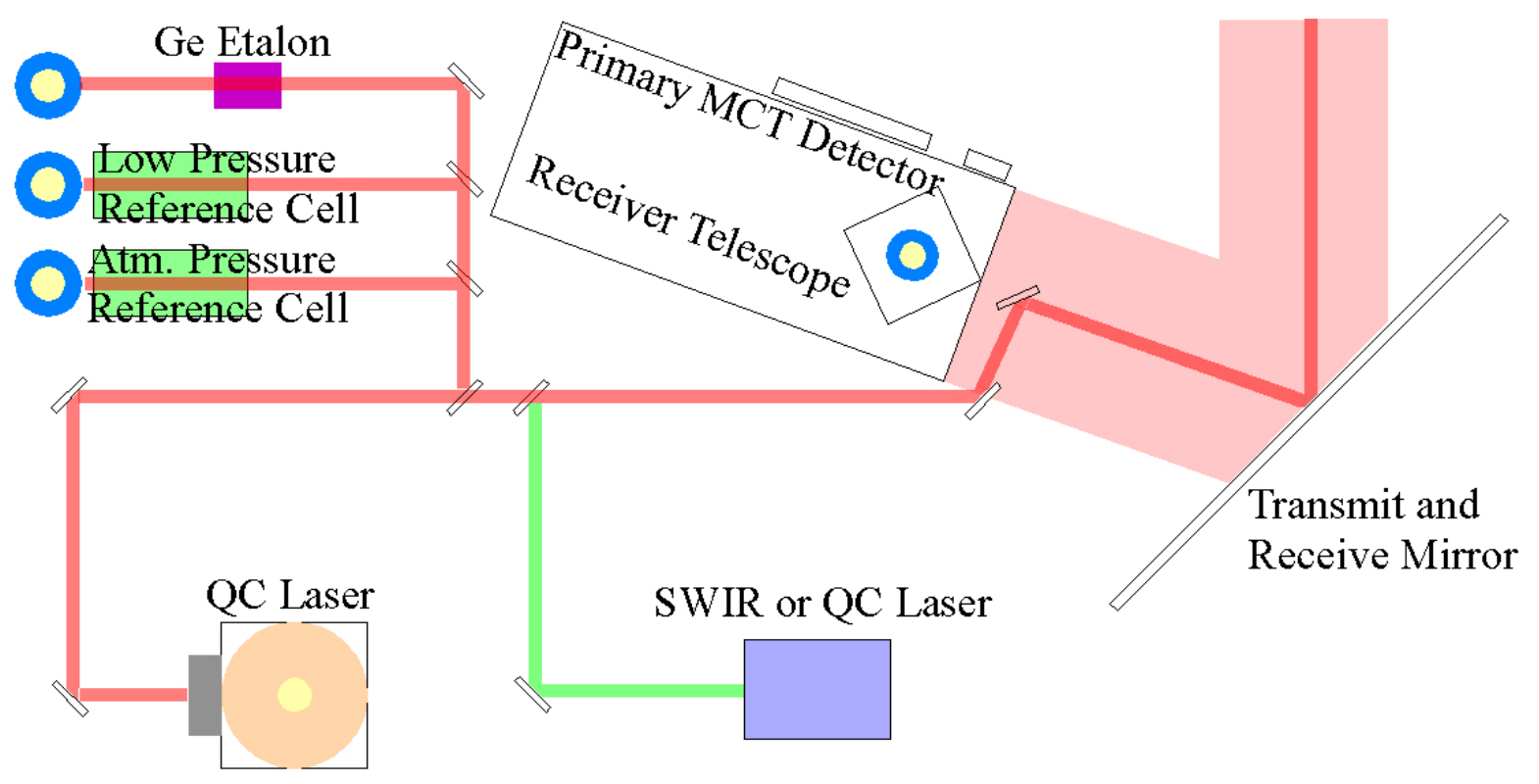

Figure 2.4. FM DIAL Optical Layout

In some experiments, discussed in Section 3, a second QC or short-wave infrared (SWIR) laser was added to the arrangement. The second QCL also operates near $8.2 \mu \mathrm{m}$ at a slightly different wavelength than the first laser. The SWIR laser is a commercial system operating at $1.56 \mu \mathrm{m}$, and is capable of outputting $20 \mathrm{~mW}$ cw power. For SWIR experiments, the primary MCT is removed and a SWIR detector element is positioned at the focal point of the primary mirror. 


\subsection{Enhancements in FM DIAL Versatility}

This section discusses the incorporation of a second laser into the FM DIAL system. In the first set of experiments an additional QC was used to simultaneously monitor two different spectral regions. The second experiment involved a comparison between a QCL operating in the LWIR and a telecommunications laser operating in the SWIR.

\subsection{Dual QCL FM DIAL Experiments}

One limitation associated with current state-of-the-art QCLs is their optical frequency (wavelength) tuning range. At present the range is less than $2 \mathrm{~cm}^{-1}$ or only about $0.15 \%$. With molecules at atmospheric pressure, which is the condition in FM DIAL experiments, even the extremely sharp features associated with small light-weight species become pressure broadened to about $0.15 \mathrm{~cm}^{-1}$ (FWHM). Accurate and unambiguous detection thus requires scanning over about $0.75 \mathrm{~cm}^{-1}$ in order to observe the full line shape. Most molecules of interest to the nonproliferation and weapons detection communities are large with high molecular weights. This means that rovibrational transitions no longer are resolvable because pressure broadening makes the individual transitions overlap to form a single, broadband contour. These band contours are typically between 1 and $50 \mathrm{~cm}^{-1}$ wide.

One way to detect large molecular species is to use a multiple wavelength approach. The basic idea behind this experiment is to obtain QC lasers that are on and off resonance of a spectral feature. The concept is similar to conventional DIAL experiments that utilize on and off resonance lasers. The multiple wavelength approach may also be used for narrow spectral features so that multiple features are detected and correlated. This would drastically reduce potential false positives and aid in the chemometric analysis.

This year we demonstrated that a multi-wavelength FM DIAL experiment can be done. We used two QC lasers at slightly different wavelengths; one at $1206.0 \mathrm{~cm}^{-1}$ (QCL \# 1), the other at $1193.5 \mathrm{~cm}^{-1}$ (QCL \# 2). The difference is $12.5 \mathrm{~cm}^{-1}$, which is 5-10 times the scanning range of an individual laser. The output from the two lasers was combined using a zinc selenide beam combiner, and transmitted to a retroreflector in the same way as single wavelength experiments. The return beams were reflected back to the receiving telescope and imaged onto a single MCT detector element. The output of the detector was preamplified and processed with two lock-in amplifiers. The first laser was modulated at $200 \mathrm{kHz}$, while the second one at $250 \mathrm{kHz}$. The lock-in amplifier was adjusted to have $6.25 \mathrm{kHz}$ bandwidth, so that the frequency channels were well resolved. Figure 3.1 shows the result of the experiments. 


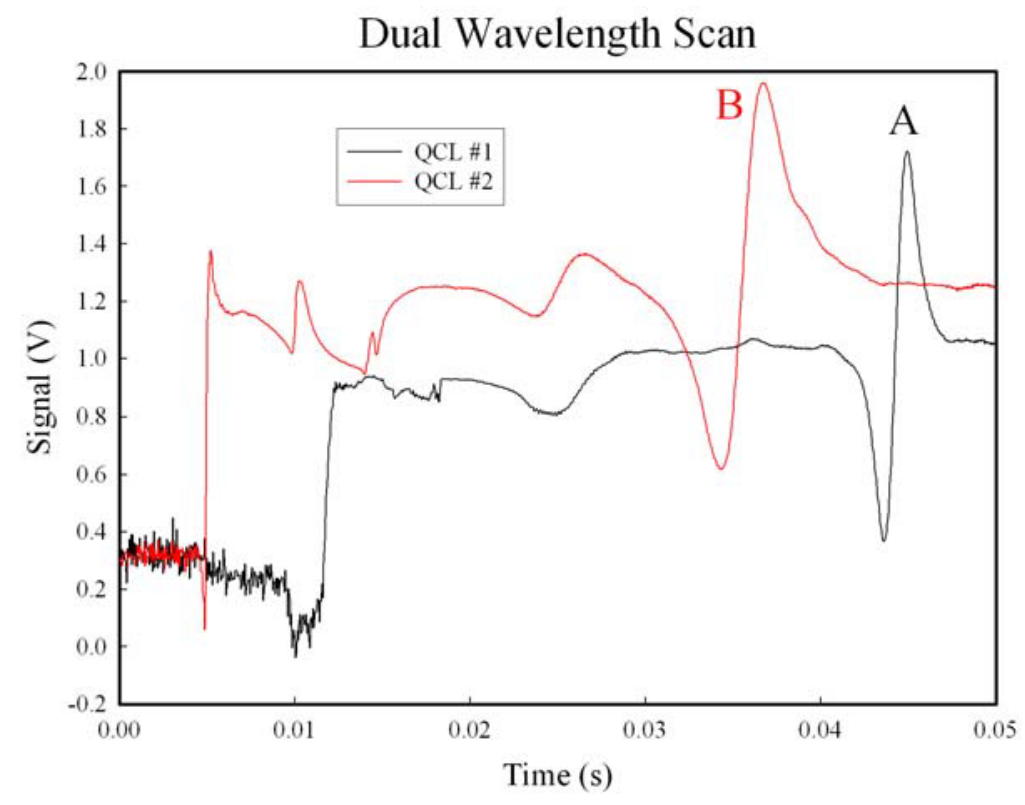

Figure 3.1. Data that was Collected from Two QC Lasers Simultaneously. The large feature at $0.35 \mathrm{~s}$ in the red trace is at $1206 \mathrm{~cm}^{-1}$, and the one in the black trace is at $1193.5 \mathrm{~cm}^{-1}$. No interferences were observed between channels.

Two current controllers were used to drive each laser simultaneously. Because two different versions of current controllers were used, one laser (QCL \#1, black trace) had a larger optical frequency sweep than the other laser. This makes the water absorption feature (A) appear narrower than that in the other trace (B). The derivative line shapes are due to ambient water absorption. There is no interference observed between channels and the data is simultaneous and independent. This detection approach could easily be used to reduce the false positive rate and enhance the detection limit, by looking for correlated signals from each laser, or be used to detect broad spectral features.

\subsection{Addition of SWIR Detection Capability}

We also added a short wave infrared laser (SWIR) and detector to the FM Dial optical system to allow for the detection of additional chemicals that are not in our QCL's tuning range and to compare and quantify the FM DIAL experiment's LWIR and SWIR performance as a function of turbulence. The additional SWIR system, lasing at $1.56 \mu \mathrm{m}$, is based on a laser diode operating in an external cavity arrangement. The output power of the laser is $20 \mathrm{~mW}$, which is significantly less than the QCL system; however, this is offset by better detectors in the SWIR and much lower background noise levels. The laser has two wavelength selective elements: a grating for coarse tuning, and an end mirror mounted on a PZT for fine-tuning. The grating can tune the output of the laser from $6369-6579 \mathrm{~cm}^{-1}$. The PZT may be used to perform frequency modulation experiments, and rapid tuning of the laser. The mirror/PZT movement limits the maximum frequency modulation that can be used to about $2-3 \mathrm{kHz}$. The grating typically scans the laser more slowly over the entire tuning range. For the FM DIAL experiments we used the grating tuning to locate a spectral absorption feature, and then performed FM experiments using 
the PZT to scan and modulate the laser's frequency (wavelength). The PZT tunability of the SWIR system is about $1 \mathrm{~cm}^{-1}$, which is slightly narrower than the QCL system. Currently we are exploring using rapid grating control, which should extend the scan range to nearly $10 \mathrm{~cm}^{-1}$ in the same amount of time as the PZT scan. 


\subsection{FM DIAL Noise Reduction}

Several different noise reduction techniques were explored and implemented in FY03. We begin with a discussion of error propagation in the FM DIAL system and continue with description of two data analysis techniques, single beam noise subtraction and intensity normalization and weighted averaging, that can significantly improve the signal to noise ratio. This is followed by experimental results obtained during the four field experiments conducted during FY03.

\subsection{Understanding Error Propagation in FM DIAL}

As outlined in the introduction section, in FM DIAL the absolute absorbance is calculated by dividing the FM optimized signal by the orthogonal RAM signal. Uncertainties in this value principally stem from two sources: intensity noise from the laser and intensity noise from turbulence. In most cases the uncertainty can be mitigated by performing a novel single beam intensity normalization procedure that we have pioneered for the FM DIAL experiment. Additional processing with a weighted averaging protocol we have developed exclusively for the FM DIAL experiment can also significantly improve the absorbance measurement confidence, especially in cases when data is collected under conditions of strong turbulence. We discuss both of these data manipulation techniques in detail below. Prior to this however, we outline the mathematical basis for deciding when it is appropriate to implement these specific noise reduction schemes.

In FY02 we worked out a derivation that shows how errors propagate from noise in the observed FM and RAM signals into the ratio of FM/RAM. We introduced two variables, $f$ and $\Delta f$, which are defined as the ratio and the uncertainty, respectively and are given by;

$$
f=\frac{a \pm \Delta a}{b \pm \Delta b} \text { and } \Delta f=\sqrt{\left(\frac{1}{b}\right)^{2}(\Delta a)^{2}+\left(\frac{a}{b^{2}}\right)^{2}(\Delta b)^{2}} .
$$

In these equations, $a$ is the FM data and $b$ is the RAM data. $\Delta a$ and $\Delta b$ are the noise in the $a$ and $b$ measurements. The ratio is denoted by $f$ and the uncertainty of the ratio is given by $\Delta f$. These variables form the basis for deciding if and how observed traces should be treated by a novel single beam intensity normalization procedure that we have pioneered for the FM DIAL experiment. There are three predominating cases; Figure 4.1 shows the simulated raw FM and RAM intensity data, and $\Delta f$ values representative for each scenario.

Case 1 occurs when the FM noise dominates, i.e., $\Delta a^{2}>>\Delta b^{2}$ (or more rigorously $b^{2} \Delta a^{2}>>a^{2} \Delta b^{2}$ ). Here the uncertainty of the ratio is dominated by the uncertainty in $a$, i.e., $\Delta f \approx \Delta a / b$. After taking the ratio, which is used to compute the absorbance, the signal-to-noise $(\mathrm{S} / \mathrm{N})$ is the same as that of the $a$ data. This is the intuitively normal case, where no unusual behavior is observed.

Case 2 occurs when RAM noise dominates, i.e., $\Delta a^{2}<<\Delta b^{2}$ (or more rigorously $b^{2} \Delta a^{2}<<a^{2} \Delta b^{2}$ ). Here the uncertainty of the ratio is dominated by $\Delta b\left(\Delta f \approx a \Delta b / b^{2}\right)$. This case is somewhat unusual because the 
noise levels in the ratio trace change depending on the magnitude of a. The uncertainty $\Delta f$ drops linearly with $a$; however as $a$ goes to zero $\Delta f$ goes to a limiting value of $\Delta a / b$. The effects are easily seen in Figure 4.1, where in the Case 2 ratio plot, different noise levels are seen at the top and bottom of the ratio peak. Also at the bottom of the figure, the uncertainty decreases significantly on the lower portion of the derivative lineshape. Interestingly, the high side of the lineshape has a corresponding ratio that has significantly larger uncertainty. These results give significant insight as to where experiments should be performed on lineshapes where only a portion of the line is accessible due to tuning range constraints. In fact, for this simulation the difference between the uncertainties for the high and low sides of the derivative lineshape, was about a factor of 200 (this is twice the ratio of $\Delta b / \Delta a$ ).

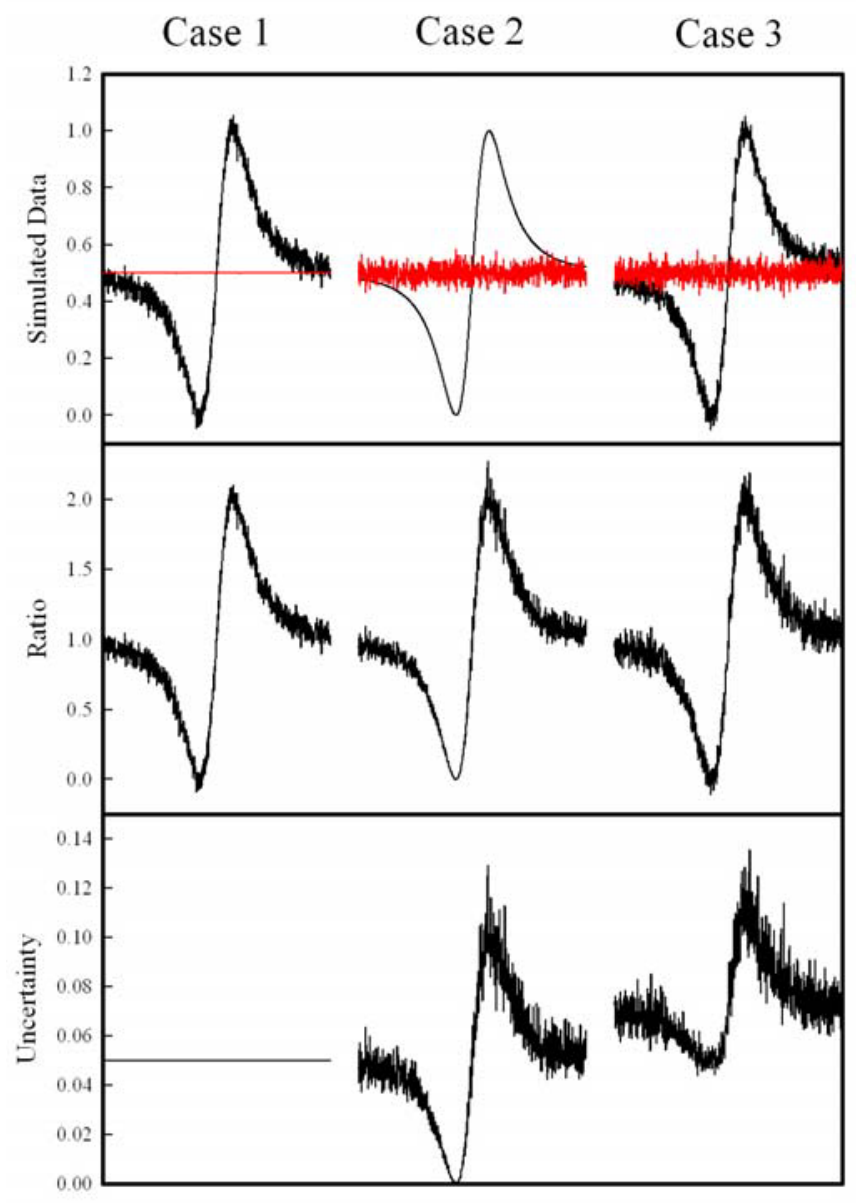

Figure 4.1. Plots of the FM (black) and RAM (red) Intensity Data (upper trace), the Ratio $f$ (middle trace), and the Uncertainty $\Delta f$ (lower trace) for the Three Cases Just Outlined in the Text

Case 3 occurs when the noise levels on both signals are equal, i.e., $\Delta a^{2} \approx \Delta b^{2}$ (or more rigorously $b^{2} \Delta a^{2}$ $\approx a^{2} \Delta b^{2}$ ), and is most appropriate for the signals we observe. This case is intermediate between case 1 and case 2 . Here $\Delta f$ may be simplified to $\Delta f \approx \Delta a\left[\left(a^{2}+b^{2}\right) / b^{4}\right]^{1 / 2}$. As seen in Figure 4.1 , there is still a minimum in the uncertainty (a factor of $\sqrt{5}$ better) for the ratio at the low side of the lineshape. It is 
interesting to note that these relative uncertainties were observed in early experiments and are now properly utilized.

In this discussion, two effects have been left out to simplify the arguments and results. The first is the slight reduction in intensity of the normalization trace due to absorption of light near the line center. This is observed in RAM traces when the peak absorbance is on the order of 0.1 and larger. This effect is insignificant for weaker absorbances, which is where most of our experimental efforts are directed. A simple extension of our derivation can be made in order to treat noise in the case of strong absorption. The second simplification was to exclude atmospheric turbulence noise. The exclusion of turbulence does not impact the above conclusions because the turbulence, which has a long time constant relative to the time required to record an FM DIAL trace, is common to both traces and normalizes out. The resulting uncertainty of the ratio is always inversely proportional to the amount of received power and the calculated uncertainty always reflects the reduction in the received power.

The careful observer will notice that in Case 3 of Figure 4.1, ratioing produces a data set with a slightly degraded signal to noise. Off the absorption line, the signal to noise is degraded by $\sqrt{2}$, while at the upper part it is degraded by $\sqrt{5}$ and at the lower part is not degraded. These observations, which were investigated, detailed and explained above, bring into question whether intensity normalization should be used, and if so, when. The decision is made by comparing the observed intensity noise with the premeasured, high frequency detector noise. If the intensity noise is less than the detector noise, then intensity normalization will degrade the signal to noise and so should not be used. If the intensity noise is greater than the detector noise, single beam intensity normalization will greatly enhance the experimental signal to noise. In nearly all of the experiments we have conducted to date, the intensity noise has been significantly greater than the detector noise.

\subsection{Single Beam Noise Subtraction and Intensity Normalization}

Theoretical research completed in FY02 outlined several experimental and data analysis procedures that could be implemented in order to measure absolute absorbance using FM DIAL, and to improve the detection limit by improving the signal to noise ratio. In FY03 we incorporated these procedures into the experiment and made measurements that confirmed their validity. The results made us confident enough to file an invention patent on our data collection and manipulation procedures. We call this invention single beam noise subtraction and intensity normalization. Here we detail its implementation into the FM DIAL experiment, and outline the experimental results.

Laser absorption techniques are powerful methods for detecting chemicals. The simplest configuration uses laser light, a sample, and a detector. Comparing transmission signals between a wavelength resonant with a chemical absorption and a wavelength that is detuned from the absorption give a signal that allows chemical concentrations to be determined. One common problem with this technique is intensity noise from the laser source. The simplest way to eliminate this effect is to add a second detector and use a double beam configuration. The second reference detector monitors the laser power only, and is used to account for any observed intensity fluctuations from the laser. This type of noise cancellation requires that the two detectors have identically matched frequency responses and that 
the signal and reference beam traverse similar paths excepting that one contains the chemical to be detected. This is an expensive way to implement noise reduction, and in some cases, such as with remote sensing, it is impractical because there is only one beam path possible. Remote sensing experiments also suffer from atmospheric turbulence, which can cause significant fluctuations in the received laser power. Often these fluctuations are the limiting noise source and seriously degrade concentration measurements.

Single beam noise subtraction and intensity normalization use a single beam path to carry both the signal and reference beams. The technique also uses a single detector but is still capable of eliminating noise due to intensity fluctuations caused by laser intensity noise, atmospheric turbulence, and scattering from particulates and fog. The basic idea behind our invention is a detection scheme that uses lock-in detection of a current modulated laser source. Quantum cascade lasers (QCL) have been used for these proof-of-concept experiments; however other lasers such as lead salt lasers and diode lasers have similar properties and so can be used for this type of spectroscopy. The experimental setup is shown in Figure 4.2; a current controller (or other device) is used to slowly scan a laser over an absorption feature, while a sine wave modulates the laser at a much higher frequency (modulation/scan $\geq 1000$ ).

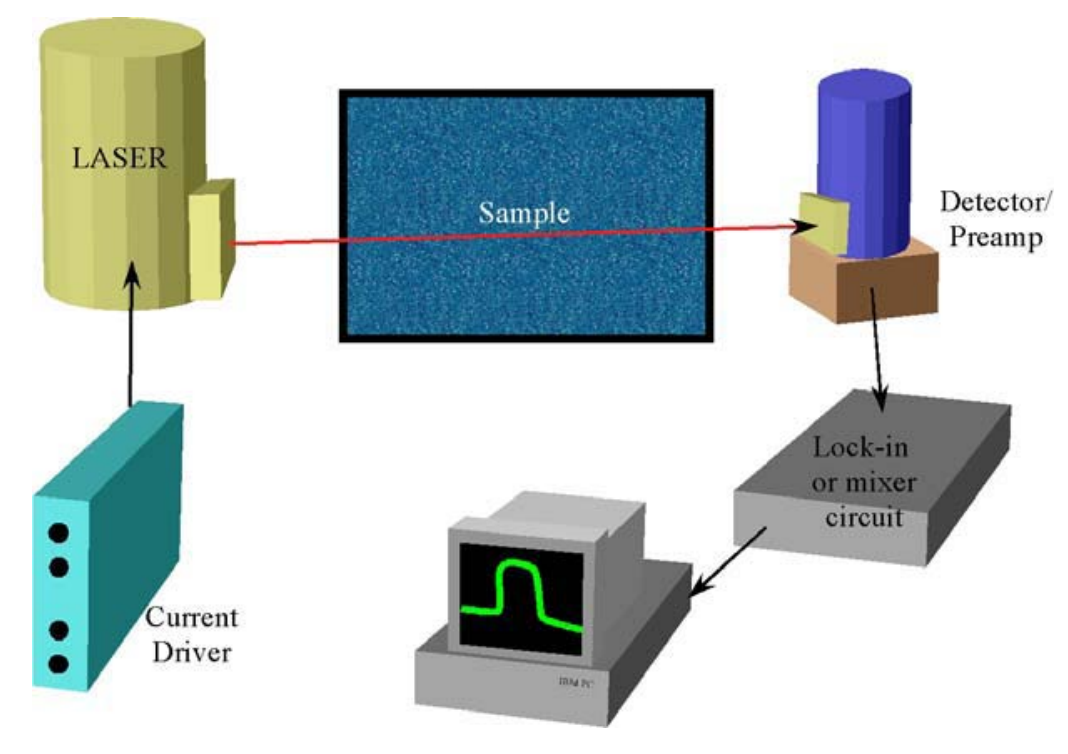

Figure 4.2. Single Beam Experimental Setup for Lock-In Detection

The laser beam is directed through a sample that has an absorption feature in the laser tuning range. The detector monitors the laser modulation and produces a signal that is sent to the lock-in amplifier. A property QCLs and diode like lasers share is that their modulated output has a frequency modulated (FM) component and an undesired amplitude modulated (AM) component. At low modulation frequencies, the FM and AM occur with the same phase. Above a characteristic frequency however the FM phase starts to lag behind the AM phase. For the QCL in use, this phase shift occurs around $200 \mathrm{kHz}$, where there is approximately $45^{\circ}$ phase lag of the FM behind the AM. This phase lag is likely due to the fact that the amplitude modulation is controlled directly by the injection current, while thermal effects in the laser control the frequency modulation. Figure 4.3 presents a simulation that represents the signals observed in an FM experiment. 


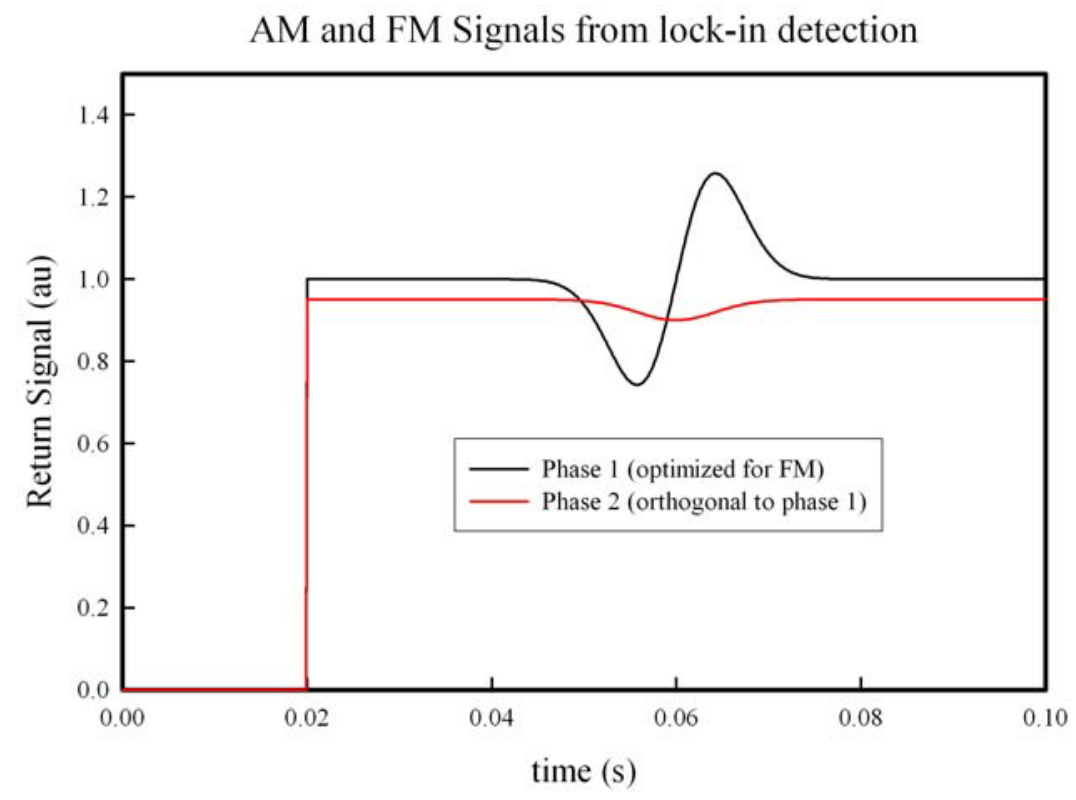

Figure 4.3. AM and FM Components. Note that while the $\mathrm{x}$ axis is given in units of time, this really represents the laser frequency (or wavelength) since the injection current is slowly being ramped upward in time.

Because there is only a $45^{\circ}$ phase shift between the AM and FM signals, the trace optimized for FM still contains a portion of the AM signal. The orthogonal trace contains information about only the AM component, as seen in Figure 4.3. Note that the AM signal shows up predominately as an offset in the figure, and the FM part shows up as the positive and negative going peak. A small negative dip at the line center is evident in phase 2 and is due to the fact that the chemical has absorbed a small amount of the transmitted light. Typically, for sensing applications this dip is much less than the offset $(<1 \%)$ and may be either ignored for weak absorptions or properly taken into account for strong absorptions. Any intensity fluctuations will show up equally in the two phases and forms the basis for the noise reduction technique. Taking the ratio of the two traces (FM/AM) eliminates intensity noise contributions. This is fairly easy to implement experimentally since the lock-in amplifier can monitor these two channels simultaneously.

The single beam noise subtraction and intensity normalization procedure was tested by placing variable attenuators on the outer rim of a beam chopper, which in turn was placed partially in the beam path. Operating the beam chopper caused the beam intensity to change in time. A trace of the resultant FM and RAM (denoted as orthogonal) signals are presented in Figure 4.4. 


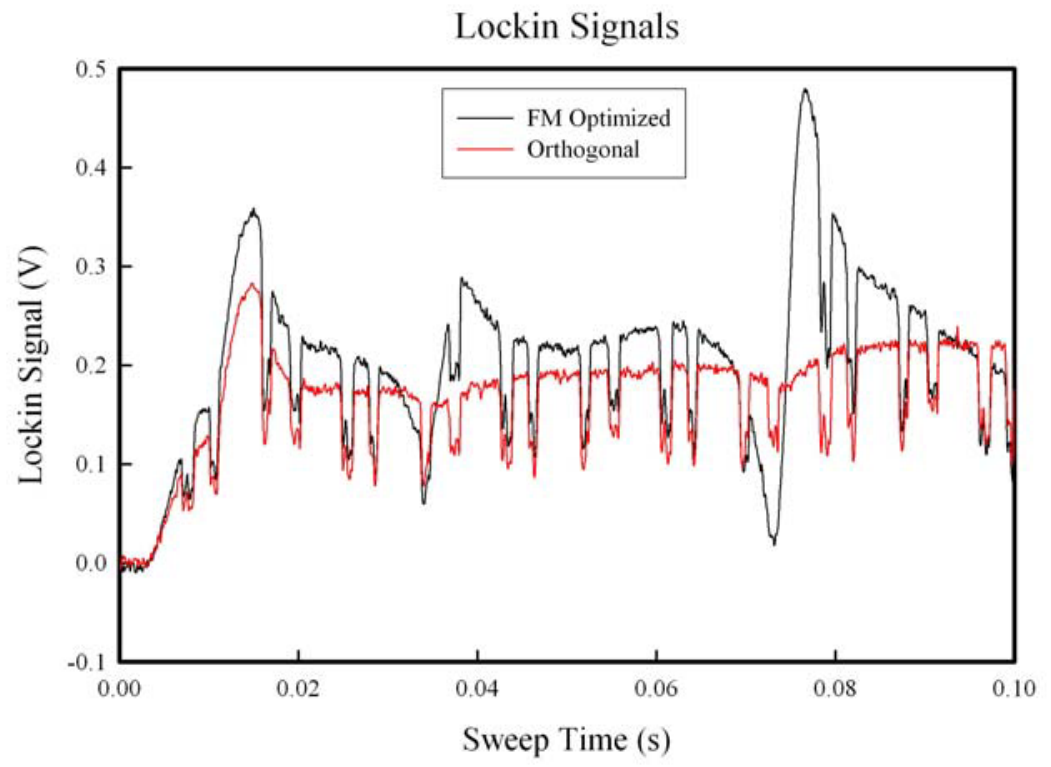

Figure 4.4. FM and RAM (orthogonal) Lock-In Signals Observed When a Mechanical Chopper is Placed in the Laser Beam Path

Note that the intensity noise is observed in both traces. Figure 4.5 shows the result of taking the ratio of the two traces from Figure 4.4.

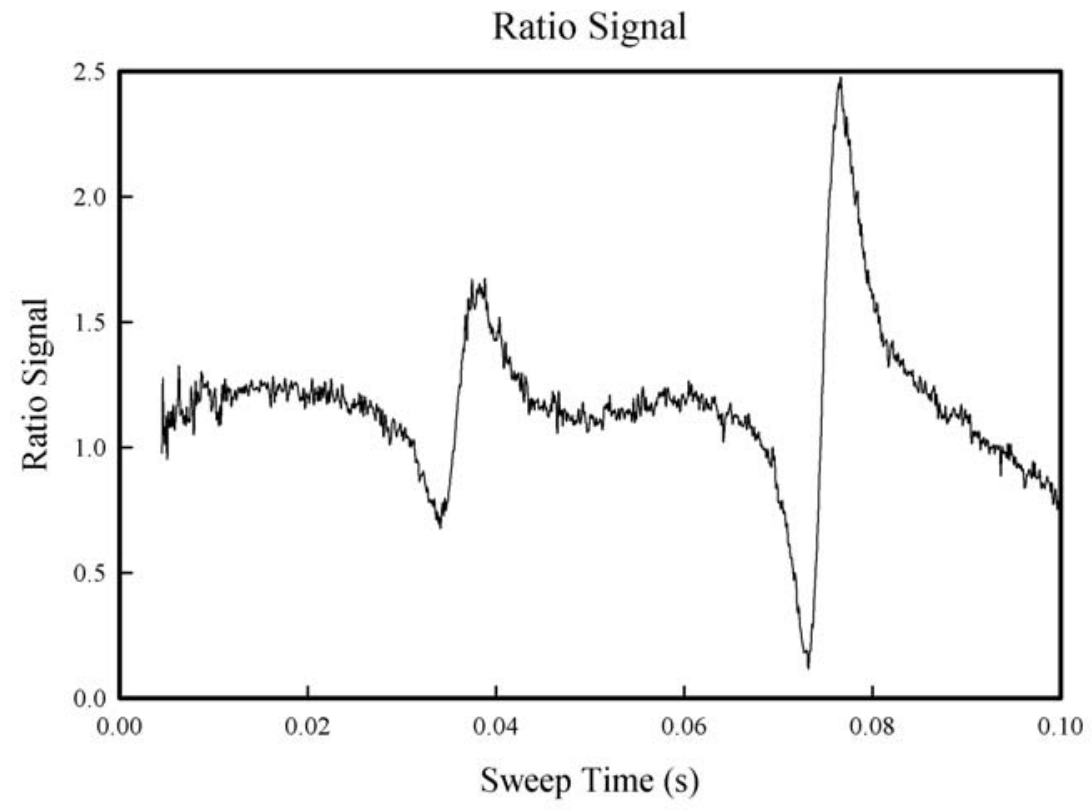

Figure 4.5. Ratio of the FM and RAM Traces Shown in Figure 4.4 
Note how the intensity noise fluctuations are eliminated. The residual noise is due to the experimental shot noise observed in Figure 4.4. This noise becomes slightly larger after taking the ratio, because it is not correlated between the two traces. Since the shot noise is constant throughout the scan, when dividing by intensity-reduced signals, the signal to noise is reduced for the ratio. This reduction in signal-to-noise is not due to the use of the ratio method, but rather reflects the fact that the signal to noise is proportional to the signal level. With additional processing, the signal to noise of each point along the trace may be estimated and is useful when combining multiple traces, or least squares fitting.

This detection scheme has numerous advantages over existing methods. One of the most important advantages is that dual beam noise reduction can be realized with a single beam system. This reduces cost and complexity by only having a single detector channel. This is a major advantage when doing remote sensing, where a reference beam is not available for noise subtraction schemes. Another advantage is that the signals from the two orthogonal phases (effectively signal and reference in the same beam) traverse the same optical path, and are detected on the same detector. This allows for efficient noise subtraction, and eliminates the need to carefully match two detectors frequency response functions. One situation where this technique does not provide any benefit is when there is little or no amplitude noise to eliminate, i.e., case 1 outlined above. Here taking ratios of the FM and AM signals gives a result that is slightly noisier than just the FM signal itself.

\subsection{Weighted Averaging}

One of the performance limiting aspects of remote sensing with lasers is noise caused by atmospheric turbulence. Turbulence causes significant fluctuations in the received power. Figure 4.6 illustrates the problem of atmospheric turbulence noise. The top panel shows a $20 \mathrm{sec}$ series of data where a constant power laser beam was transmitted $2.25 \mathrm{~km}$ to a retro reflector, and the received beam was detected with a 10 " f-3 receiving telescope. The bottom panel expands the first $0.2 \mathrm{~s}$ of data, and shows how the received signal fluctuates. Notice that at certain times the signal is strong $(0.9 \mathrm{~s})$ and sometimes the signal is completely lost $(0.17 \mathrm{~s})$.

This variability can strongly influence the performance of remote sensing systems, and must be treated appropriately. Intensity normalization can remove much of these effects, and was discussed previously. However, in addition to normalization, one can also perform a certain type of data averaging to maximize the signal to noise.

The general definition of an average (or mean) is given by,

$$
M_{r}=\left[\sum_{i=1}^{N} w_{i} x_{i}^{r}\right]^{1 / r},
$$

where $N$ is the number of measurements, $w_{i}$ are the normalized weighting factors, $x_{i}$ are the data, and $r$ is an integer. Different values of $r$ correspond to different ways to average, for instance when $r=1$ the result is a standard mean. Some other possibilities are $r=2$ (quadratic mean), $r=0$ (geometric mean), $r=-1$ 

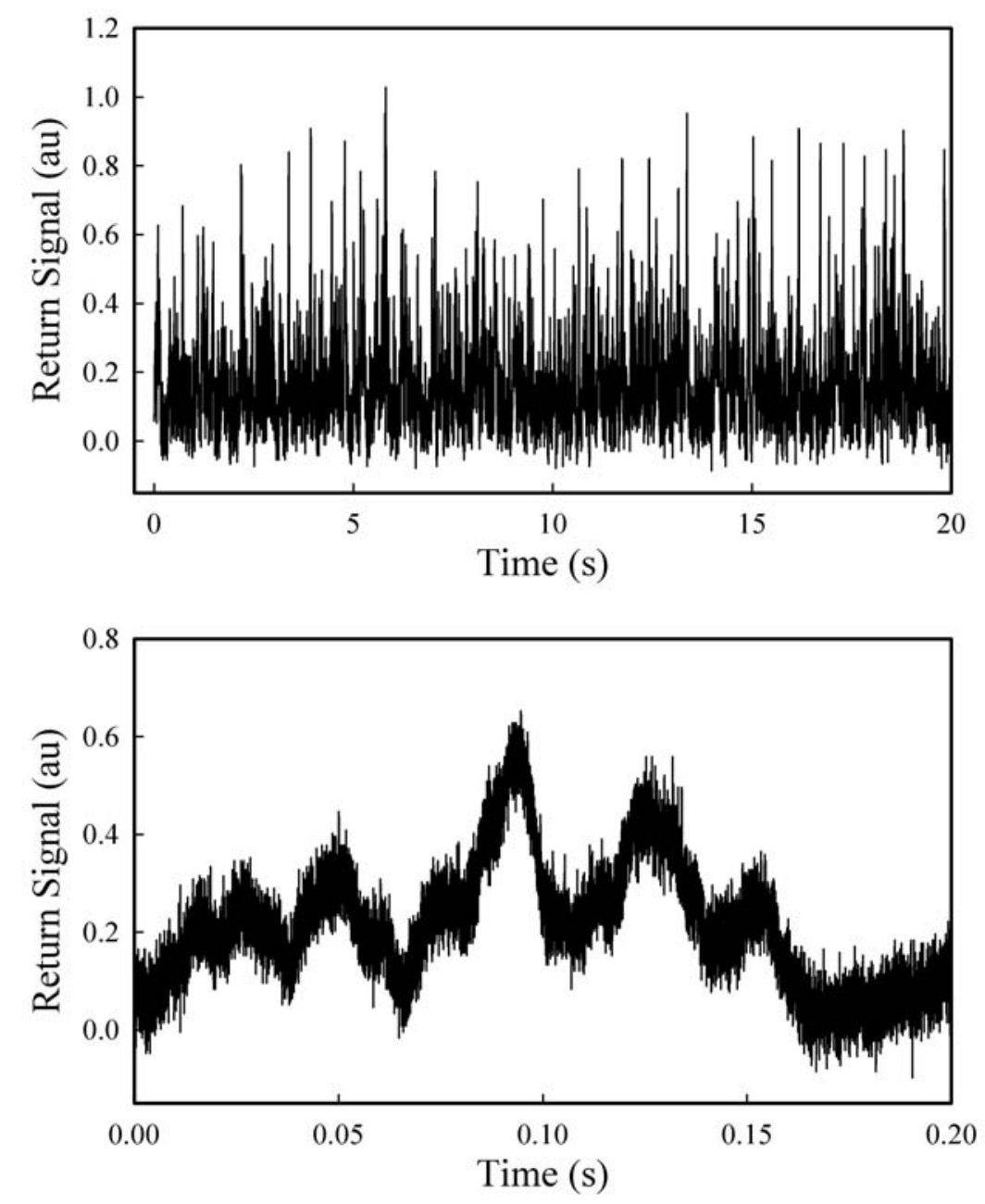

Figure 4.6. Influence of Atmospheric Turbulence on FM DIAL Returned Intensity

(harmonic mean). For our measurements, $r=1$ is the most appropriate form of averaging and the equation simplifies to the following;

$$
M_{1}=\frac{1}{W} \sum_{i=1}^{N} w_{i} x_{i} \quad, \quad W=\sum_{i=1}^{N} w_{i}
$$

In these equations, the possibility for un-normalized weights has been included $(W \neq 1)$. There is still the choice to pick any set of weighting coefficients, $w_{i}$, to form the mean. Typically, these are set equal to unity and the formula simplifies to summing all elements and dividing by the number of measurements (simple mean), i.e.,

$$
\langle x\rangle=\frac{1}{N} \sum_{i=1}^{N} x_{i}
$$


The uncertainty of the mean can be calculated from the uncertainties of the data points using propagation of errors. Propagation of errors reveals that the uncertainty is

$$
\Delta\langle x\rangle=\frac{1}{N}\left[\sum_{i=1}^{N} \Delta x_{i}^{2}\right]^{1 / 2} .
$$

The major problem with using this type of averaging is that all data is treated equally. In the case where each data point has a different uncertainty, as with our FM DIAL data, simple averaging is not very effective. The problem is that the uncertainty of the mean is dominated by the largest $\Delta x_{i}$, which corresponds to the worst measurement. The mean is also affected because both the poor and good measurements contribute equally to the average.

A solution to this problem is to use a weighted mean where the $w_{i}$ are picked to have useful properties. By picking the weights as

$$
w_{i}=\frac{1}{\Delta x_{i}^{2}},
$$

the mean becomes

$$
\langle x\rangle_{\text {weighted }}=\frac{\sum_{i=1}^{N} \frac{x_{i}}{\Delta x_{i}^{2}}}{\sum_{i=1}^{N} \frac{1}{\Delta x_{i}^{2}}}
$$

and, using propagation of errors, the uncertainty is

$$
\Delta\langle x\rangle_{\text {weighted }}=\frac{1}{\sqrt{\sum_{i=1}^{N} \frac{1}{\Delta x_{i}^{2}}}} .
$$

The major advantage of using this form of weighted averaging is that now the uncertainty of the mean is dominated by the best measurement (smallest $\Delta x$ ). Also, the weighted mean is insensitive to measurements that have large errors because the term $x_{i} / \Delta x_{i}^{2}$ becomes very small. This form of averaging gives the minimum variance estimate of the average and is the most appropriate way to treat data with unequal uncertainties.

To validate the weighted form of averaging we performed several simulations to test different forms of averaging. The simplest simulation involved generating sequences of normally distributed random numbers and calculating the mean and uncertainty for several types of averaging. As an example, the data set shown in Figure 4.7 was used for comparison of different averaging techniques. 


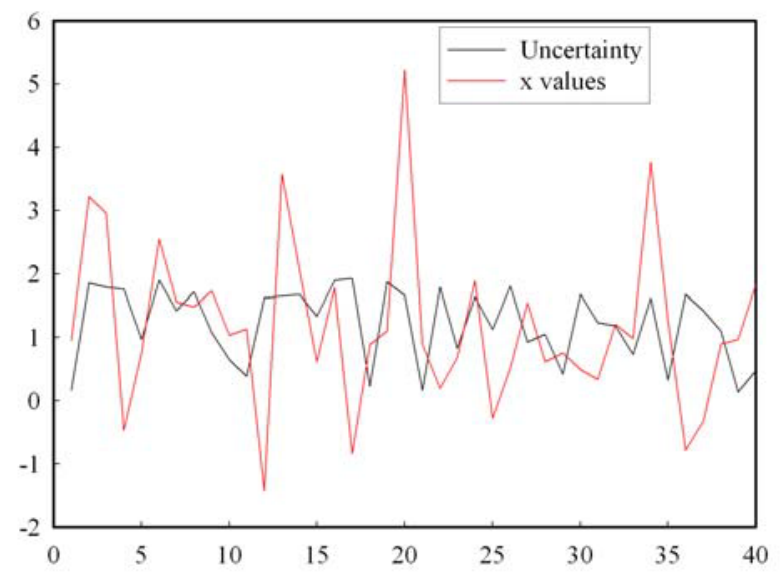

Figure 4.7. Data Set Used in Averaging Technique Comparison

The uncertainties (in black) were generated to represent data with different uncertainties ranging from 0.1 to 2.0. These uncertainties were then used to generate $\mathrm{x}$ values randomly selected from a normal distribution with mean of one. Performing averaging with different assumptions for the weighting coefficients gave the results that are listed in Table 4.1. As can be seen the simple mean with equal weights actually has the worst signal to noise and is the furthest from the true value. The best result is obtained for the case where the weights are $1 / \Delta x^{2}$. The standard mean has $18.4 \%$ error, with a signal to noise of 5.5, and the true value is within the uncertainty of the mean. In comparison, the weighted mean $\left(1 / \Delta x^{2}\right)$ has only $-1.0 \%$ error, a signal to noise of 13.5 , and again the true value is within the uncertainty of the mean. For this case the error of standard averaging was about 18 times worse than the weighted average.

Table 4.1. Weighted Mean Comparison. The $\mathrm{S} / \mathrm{N}$ column is the signal to noise ratio for the mean (mean/uncertainty).

\begin{tabular}{||c|c|c|c|c||}
\hline $\begin{array}{c}\text { Weighting } \\
\text { Coefficient }\end{array}$ & $\begin{array}{c}\text { Weighted } \\
\text { Mean }\end{array}$ & Uncertainty & \% Error & S/N \\
\hline 1 & 1.1839 & 0.2136 & 18.4 & 5.5 \\
\hline $1 / \Delta x$ & 1.0709 & 0.1101 & 7.1 & 9.7 \\
\hline $1 / \Delta x^{2}$ & 0.9895 & 0.0734 & -1.0 & 13.5 \\
\hline $1 / \Delta x^{3}$ & 0.9596 & 0.079 & -4.0 & 12.2 \\
\hline $1 / \Delta x^{4}$ & 0.9502 & 0.0843 & -5.0 & 11.3 \\
\hline $1 / \Delta x^{5}$ & 0.9479 & 0.0883 & -5.2 & 10.7 \\
\hline $1 / \Delta x^{6}$ & 0.9481 & 0.0918 & -5.2 & 10.3 \\
\hline $1 / \Delta x^{7}$ & 0.9493 & 0.095 & -5.1 & 10.0 \\
\hline $1 / \Delta x^{8}$ & 0.9508 & 0.0982 & -4.9 & 9.7 \\
\hline $1 / \Delta x^{9}$ & 0.9524 & 0.1014 & -4.8 & 9.4 \\
\hline $1 / \Delta x^{10}$ & 0.9539 & 0.1047 & -4.6 & 9.1 \\
\hline
\end{tabular}


A second, more realistic, simulation extended these results to approximate the results of averaging multiple FM DIAL traces together. Here ten sets of data were generated; each consisting of 1000 points similar to the above simulation, but the standard deviations were chosen to vary in a periodic fashion. Figure 4.8 shows the standard deviations of each data point in the bottom panel, and the actual $x_{i}$ values in the top panel.

The top panel of Figure 4.9 shows the result for simple averaging in black and that for weighted averaging in red. Notice the significant improvement in the signal to noise that results when the weighted averaging scheme is used. The bottom panel of Figure 4.9 shows the uncertainties calculated for the simple average and the weighted average. From this it is clear that the weighted mean uncertainties are about five times smaller than for the simple mean.

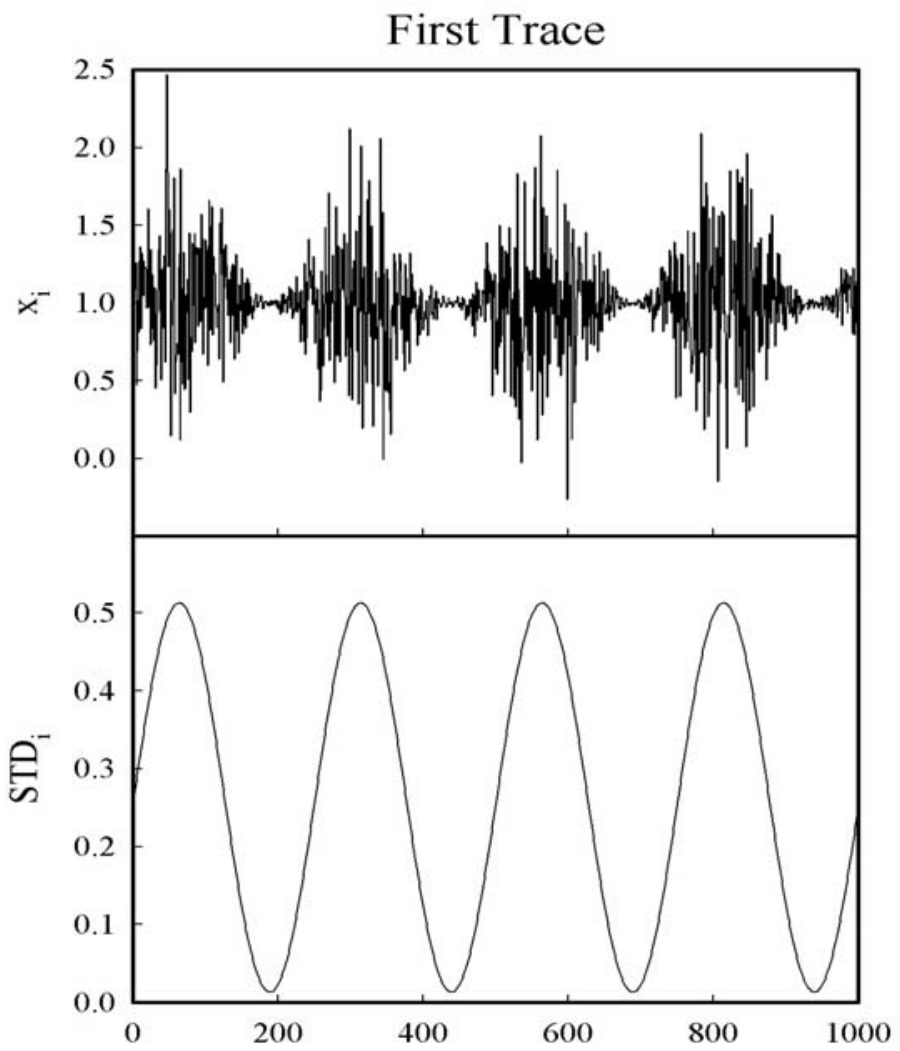

Figure 4.8. Generated Standard Deviations (bottom) that Range from 0.0025 to 0.5 . Top panel shows the $x_{i}$ values that are selected from the normal distribution using the corresponding standard deviation. 

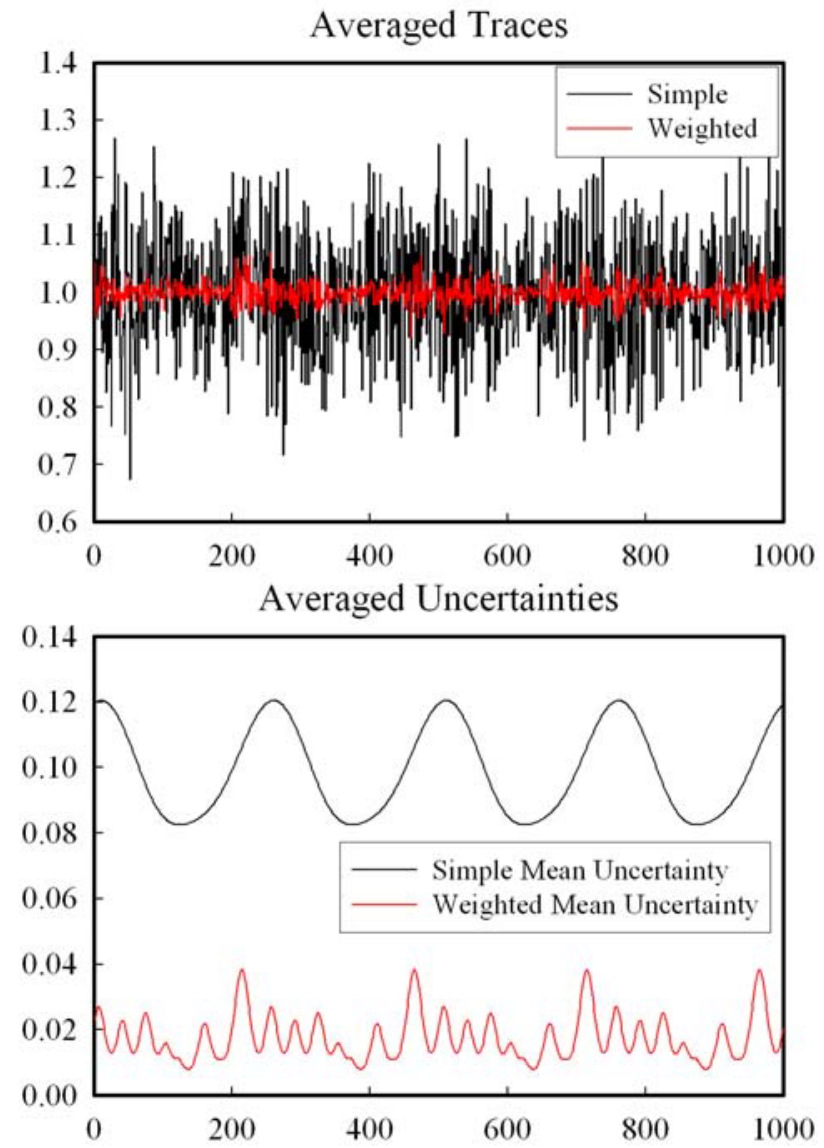

Figure 4.9. Results from Averaging 10 Different Traces

Data from field experiments conducted at the Hanford town site (HTS I) were also used to investigate the effects of averaging and validate our simulations. The FM DIAL system was deployed with a retro reflector positioned $2.25 \mathrm{~km}$ from the source. An $8.2 \mu \mathrm{m}$ laser was directed along a horizontal path to the retro and a portion of the beam reflected back to the receiving telescope and detector. After a $4.5 \mathrm{~km}$ round trip the turbulence noise was extremely bad, as can be seen in the top panel of Figure 4.10 which shows the first four traces of a block of 512 traces collected. Notice that it is impossible to distinguish any structure of an absorption features in the data. After taking a simple mean of the 512 traces, which is given by the black line in the bottom panel, the absorption features emerge, in particular at 0.03 and $0.075 \mathrm{~s}$. Taking the weighted mean gives a much better averaged trace, as depicted by the red line in the bottom panel. Here the signal to noise is enhanced by about a factor of ten. The differences between weighted and simple averaging techniques become even more pronounced during higher turbulence conditions and over longer ranges. 


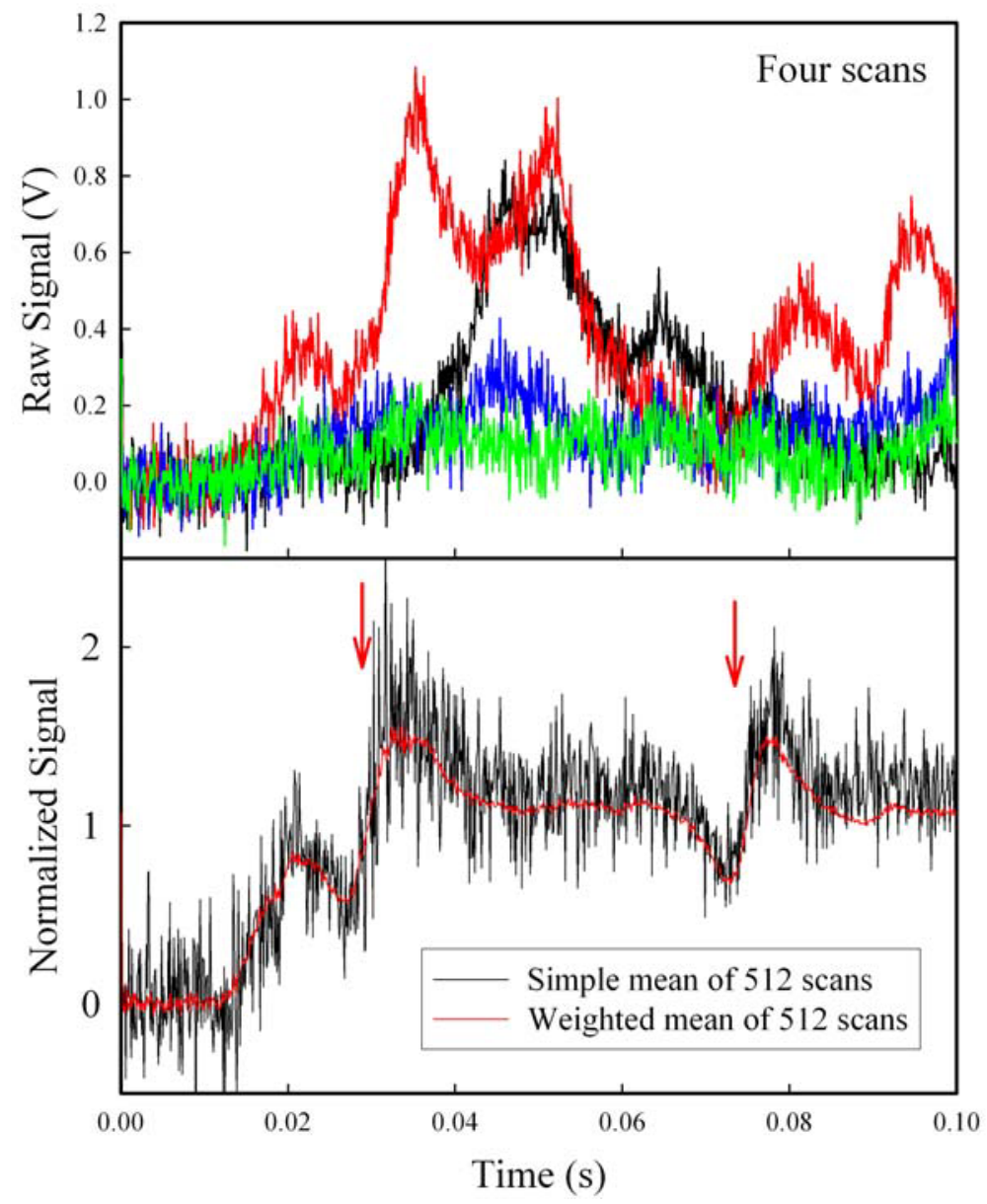

Figure 4.10. HTS1 FM DIAL Experiments. The top panel shows four representatives scans over absorption features. Structure is not visible within the turbulence noise. The bottom panel shows the result for simple (black) and weighted (red) averaging schemes, after normalization.

\subsection{Influence of Turbulence on FM DIAL Signal to Noise}

This section begins with a description of field tests performed at the Hanford Town Site in March 2003. This is followed by a discussion of atmospheric turbulence.

\subsubsection{Dependence of Range and Sweep Rate on FM DIAL Performance}

The first round of experiments at HTS in February 2003 provided some useful guidance on what should be done for this set of tests. This set of tests was designed to test the sensor performance at different ranges and different sweep rates. Two realistic sensing distances were tested, $2.23 \mathrm{~km}$ and $4.7 \mathrm{~km}$. The goal was to observe how the detection limit drops as a function of distance. Two retroreflectors were deployed, and the gimble mirror was used to conveniently switch between them. 
Selection of the ramp frequency that scans the laser is important, and potentially coupled to the distance to the retro-reflector. While slow ramps allow narrow band/low noise detection of the frequency modulation, they also collect fewer traces (spectra) for averaging and more turbulence effects are observed over the longer timescale. On the other hand, fast ramps allow many traces to be collected and atmospheric turbulence may be considered 'stationary'; however, the signal to noise of each trace is degraded because wider bandwidth detection must be used.

Different ramp frequencies were tested at 1,10,100, and $500 \mathrm{~Hz}$. These choices are bounded by practical limitations of our experiment. Typically, our data traces consist of 1000 points and the time constant of the lock-in amplifier is adjusted to match. At $1 \mathrm{~Hz}$ data is collected every $1 \mathrm{~ms}$, and the time constant is set to $1 \mathrm{~ms}$. For data collection rates slower than this, turbulence cannot always be considered stationary within the time constant used. Also, data collection rates become slow and the observed signal can change as the laser is scanned over the feature. The fast end limitation is caused by the way that we are operating the laser, and the current controller used to drive the laser. As described in last years report, the amount of frequency modulation (modulation index) drops off rapidly above $200 \mathrm{kHz}$. Optimum FM detection requires that the laser modulation is approximately equal to the FWHM of the probed absorption feature (modulation index $\approx 1$ ). For atmospherically broadened single rovibrational transitions, this is typically $0.1 \mathrm{~cm}^{-1}$ to $0.2 \mathrm{~cm}^{-1}$ ( $3 \mathrm{GHz}$ to $6 \mathrm{GHz}$ ). Above $200 \mathrm{kHz}$, the laser is no longer able to provide the optimum frequency modulation. Fortunately even at $100-500 \mathrm{~Hz}$, the atmosphere can be regarded as stationary and faster modulations are not necessary. Faster ramps are possible, however the benefits are limited. Faster ramps will provide a larger number of traces to average together (enhancing the $\mathrm{S} / \mathrm{N}$ ), but the tradeoff is that wider bandwidth detection must be used (degrading the $\mathrm{S} / \mathrm{N}$ ).

In this experiment the trailer was positioned at the HTS and the main turning mirror could be adjusted between two 6" retro-reflectors. The first retro (R1) was positioned at $2.32 \mathrm{~km}$, and the second one (R2) at $4.67 \mathrm{~km}$ on a hill. The beam path to R1 was close to ground level $(<5 \mathrm{~m})$, while the path to $\mathrm{R} 2$ was above ground level $(>10 \mathrm{~m})$ for a majority of the beam path. These differences in paths should have effects on the signal to noise levels, but this is the subject of forthcoming studies. Future experiments will place the trailer on a higher position that is capable of sighting several retro-reflectors positioned over a broad range. Ambient water vapor transitions were selected for signals so that a plume release was unnecessary. Data was collected with $\mathrm{R} 2$ for each of the ramp frequencies $(1,10,100,500 \mathrm{~Hz})$ in rapid succession, and then the R1 data was collected. Finally R1 and R2 data were collected in rapid succession using the $100 \mathrm{~Hz}$ ramp for comparison. Representative data from $\mathrm{R} 2$ at $1 \mathrm{~Hz}$, consisting of 512 traces, is shown in Figure 4.11. Notice that in the top panel the noise level is significant, and several traces have large fluctuations such as the dip at $0.4 \mathrm{~s}$. The bottom trace is the result after single beam normalization and shows how the turbulence effects are eliminated.

After normalizing, individual data traces were averaged to produce a high signal to noise trace that was used in a nonlinear least squares fitting procedure. That fitted function was then used as the basis, along with slope and offset terms, for a linear least squares fitting procedure for each individual trace. This emulates the procedure when a reference gas cell is used to determine the shape of the fitting function. The fitting procedure provided both the best-fit coefficients, and the estimated uncertainties of the fit shown in Figure 4.12. 

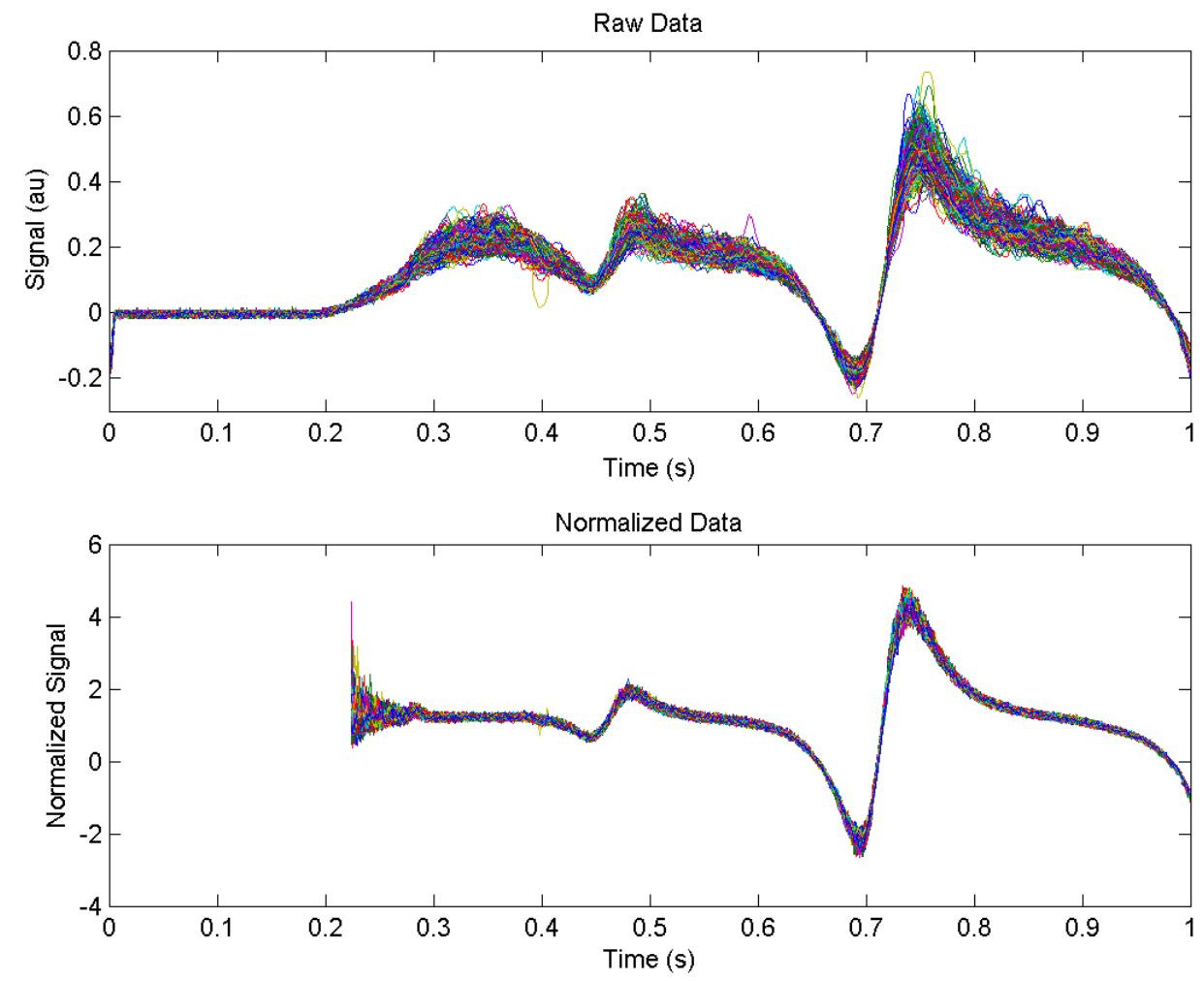

Figure 4.11. Top Panel Shows Raw Data from the $4.67 \mathrm{~km}$ Retro-Reflector, Consisting of Two Water Derivative Absorption Features. Significant noise due to atmospheric turbulence is embedded in the data. After normalization by the RAM, the bottom panel shows increased signal to noise.

The signal to noise of the experiment may be evaluated in one of several ways. We have used the parameters, and estimated uncertainties, from the linear least squares to determine the signal to noise. Propagation of error was used on the results to determine the signal to noise for the overall data set of 512 traces. The result was then scaled by dividing by the square root of the collection time to get the signal to noise for 1 second of averaging. This results in a upper limit, $\mathrm{S} / \mathrm{N}_{\text {upper }}$, and is the most favorable way to estimate the signal to noise for this type of data. We also looked at the observed fluctuations in the estimated signal over all 512 traces, and calculated the standard deviation and again scaled to 1 second of averaging. This method will produce a rigorous lower limit estimate, $\mathrm{S} / \mathrm{N}_{\text {lower }}$, of the signal to noise because any fluctuations in the water concentration will be included as noise. A better way to calculate the same quantity is use the standard deviation of the differences between successive data points and scale by $\sqrt{2}$. This provides a noise estimate, $\mathrm{S} / \mathrm{N}_{\text {intermediate }}$ that is not sensitive to water concentration fluctuations. The true signal to noise of our sensor should be somewhere between $S / \mathrm{N}_{\text {lower }}$ and $\mathrm{S} / \mathrm{N}_{\text {upper }}$. For the HTS II experiments, the calculated $\mathrm{S} / \mathrm{N}_{\text {lower }}$ and $\mathrm{S} / \mathrm{N}_{\text {upper }}$ agreed to within a factor of four. For the data shown in Figure 4.12, the $\mathrm{S} / \mathrm{N}_{\text {upper }}$ was 361 and $\mathrm{S} / \mathrm{N}_{\text {lower }}$ was 145 (for 1 second of averaging). The true signal to noise was probably closer to $\mathrm{S} / \mathrm{N}_{\text {upper }}$, because it is easily seen that the water concentration was changing in the top panel of Figure 4.12. 

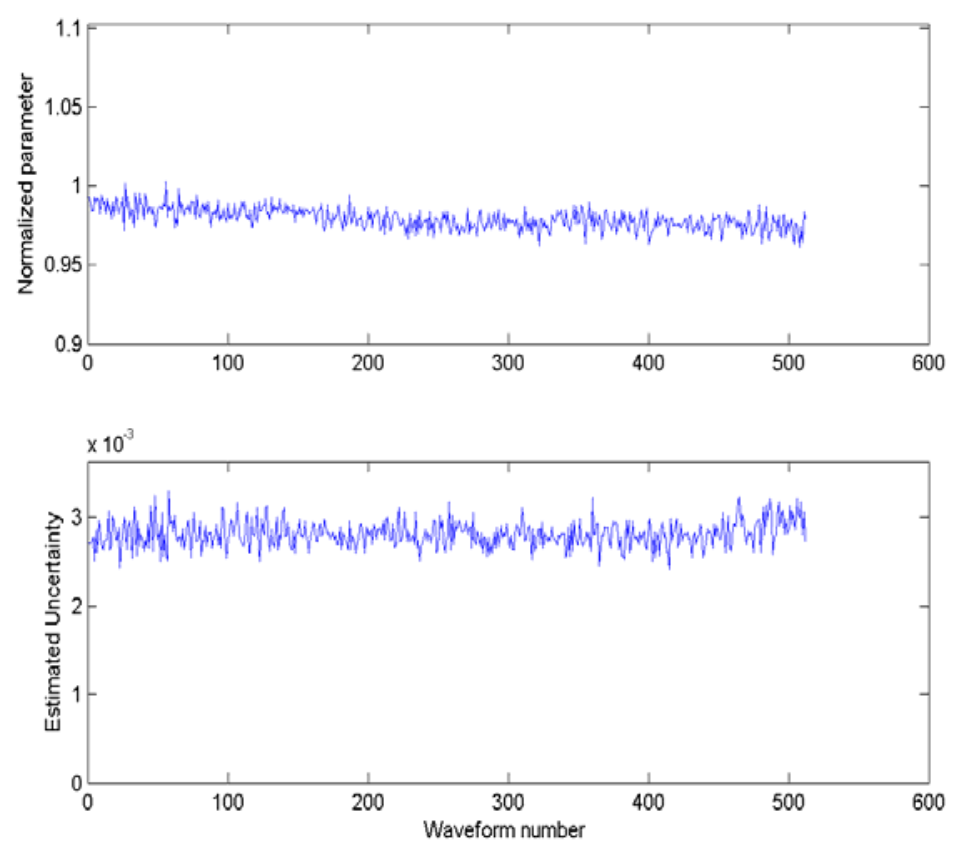

Figure 4.12. Top and Bottom Panels Show the Peak Shape Height (normalized) and the Estimated Uncertainty for Each Measurement, Respectively. The signal to noise is about 360 for each trace. Overall signal to noise is 8162 . There is a slight decrease in the water concentration (top panel) over the $512 \mathrm{~s}$ duration of measurement.

We investigated the signal to noise as a function of laser scan (or ramp) frequency. The results for the $2.32 \mathrm{~km}$ distance show that it is more favorable to ramp at faster frequencies. The $\mathrm{S} / \mathrm{N}_{\text {upper }}$ was found to be 410 for a $1 \mathrm{~Hz}$ ramp, and increased to 1730 at $100 \mathrm{~Hz}$. The $\mathrm{S} / \mathrm{N}_{\text {lower }}$ was found to go from 101 at $1 \mathrm{~Hz}$ to 816 for $100 \mathrm{~Hz}$ scans. The results for the long range $(4.67 \mathrm{~km})$ were consistent; however, the $500 \mathrm{~Hz}$ data showed a noticeable decrease in signal to noise. We attribute this to excessive high frequency detector noise that results from the wider bandwidth detection necessary for monitoring the faster signals. As the high frequency noise becomes greater than about $10 \%$ of the actual peak signals, the normalization technique tends to have limited usefulness. This limitation comes from the fact that as the dominant noise source goes from turbulence (correlated noise) to detector noise (uncorrelated) the normalization procedure starts to amplify the noise levels. Slowing the ramp frequency and narrowing the detection bandwidth such that the detector noise is significantly smaller than the return signals can always avoid this. Figure 4.13 shows the signal to noise results for the $4.67 \mathrm{~km}$ data.

Figure 4.13 shows that as the high frequency noise become more significant the $\mathrm{S} / \mathrm{N}_{\text {upper }}$ starts to suffer. From this data, it is obvious that the long-range experiment will benefit from having a reduced ramp frequency so that the data traces have high signal to detector noise. This requirement is not met for ramp frequencies faster than $100 \mathrm{~Hz}$. Data from the near retro-reflector has similar properties; however, because the return signals are significantly larger, higher frequency ramps may be used. The data indicate that the $100 \mathrm{~Hz}$ ramp is optimum for the $2.32 \mathrm{~km}$ range. 


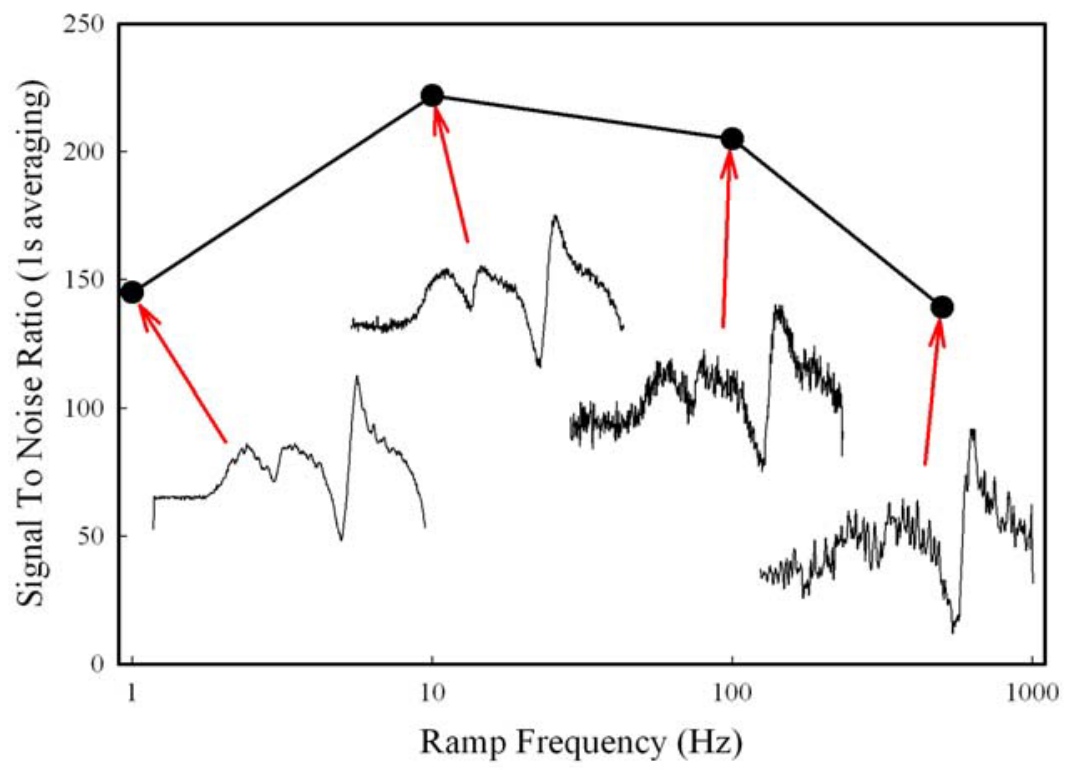

Figure 4.13. Signal to Noise $\left(\mathrm{S} / \mathrm{N}_{\text {upper }}\right)$ for the $4.67 \mathrm{~km}$ Retro (R2) are Indicated by the Circles. Representative data traces are included to show the relative effects of high frequency detector noise.

The main conclusion from these experiments is that the ramp frequency needs to be optimized according to the distance being probed. This requirement is driven by the need for high return levels compared to the detector noise. At close ranges of less than $2 \mathrm{~km}$, ramping at the maximum rate is beneficial. For long range experiments, where there are weak return levels, the detection bandwidth must be narrowed to the point that the return signals are significantly larger than $(>10 \times)$ the detector noise floor. Fortunately with the single beam normalization procedure, the additional turbulence noise that is introduced by using slower ramps is efficiently eliminated.

\subsubsection{Effects of Atmospheric Turbulence}

Atmospheric turbulence can have two effects on laser beam propagation: beam breakup and beam wander. Beam breakup occurs when the turbulence scale is smaller than the beam diameter. As light passes through turbulent regions, different portions of the beam can travel different path lengths resulting in multiple wavelets with different phases. These wavelets can constructively and destructively interfere causing a Gaussian beam shown in Figure 4.14a to fragment into a speckled pattern like that of Figure $4.14 \mathrm{~b}$. When the turbulence scale is larger than the beam diameter, beam wander occurs. Under these circumstances, the turbulence deflects or steers the beam in different directions instead of breaking it up as illustrated in Figure 4.14c. Atmospheric turbulence can occur at multiple, simultaneous scales producing patterns that are linear combinations of speckle and beam wander as shown in Figure 4.14d. 


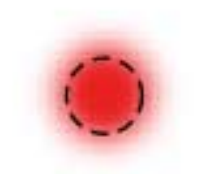

(a)

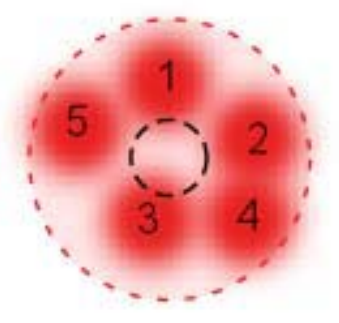

(c)

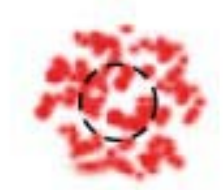

(b)

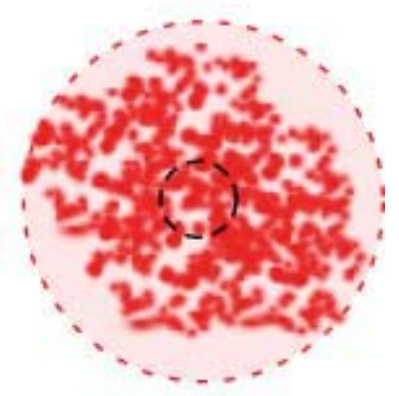

(d)

Figure 4.14. Effect of Atmospheric Turbulence on Laser Beam Footprint. (a) no turbulence, (b) turbulence induced beam breakup (c) turbulence induced beam wander at five different instants, (d) turbulence induced beam breakup and wander at five different instants.

The effect of atmospheric turbulence on signal strength depends on the relative sizes of the footprint pattern and target aperture. Consider an aperture that is comparable to the non-turbulent footprint in Figure 4.14a, depicted by the dashed black circle, for each of the four cases:

a) If the transmitter and receiver are well aligned, the signal strength will be nearly $100 \%$, but offsets in alignment will significantly reduce the signal strength.

b) The target aperture is now smaller than the footprint pattern resulting in a lower signal strength, however it will be relatively unchanged by small alignment shifts.

c) The signal strength would be negligible at each of the five instants shown, but could be nearly $100 \%$ when conditions steer the beam into the target aperture.

d) As in (b), the speckle pattern serves to average the turbulence resulting in lower, but nearly constant signal strength.

The area of the footprint pattern scales as the square of the distance between the transmitter and receiver. The QC laser has a divergence of 1.5 mrads. Without the effects of turbulence, a Gaussian beam would have a diameter of $15 \mathrm{~cm}$ at $100 \mathrm{~m}, 1.5 \mathrm{~m}$ at $1 \mathrm{~km}$ and $7.5 \mathrm{~m}$ at $5 \mathrm{~km}$. The addition of turbulence only serves to further spread and degrade the beam so that the signal strength decreases faster than the square of the distance. It should also be noted that this is only the one-way degradation. For the bistatic mode, the same processes affect the beam on its return to the telescope so that the overall signal strength can decrease by more than distance to the fourth power. 


\subsubsection{Quantifying Atmospheric Turbulence}

One method of quantifying the atmospheric turbulence is by calculating the irradiance variance or scintillation index

$$
\sigma_{i}^{2}=\frac{\left\langle I^{2}\right\rangle-\langle I\rangle^{2}}{\langle I\rangle^{2}}
$$

Here $I$ is the laser beam intensity and \langle\rangle denotes the ensemble average. Figure 4.15 shows the detector voltage as a function of time for a five second period on July 18, 2003 at 6:30 AM (blue line) and 1:06 PM (red line). In the morning, the data have a larger average and are uniformly distributed above and below the mean but in the afternoon the average is much lower with occasional periods of strong signal returns. The scintillation index quantifies these differences. At 6:30 AM the scintillation index has a value of only 0.00872 , while it is 0.495 in the afternoon - an increase of more than a factor of fifty. The retro-reflector was placed approximately one kilometer away at both times.

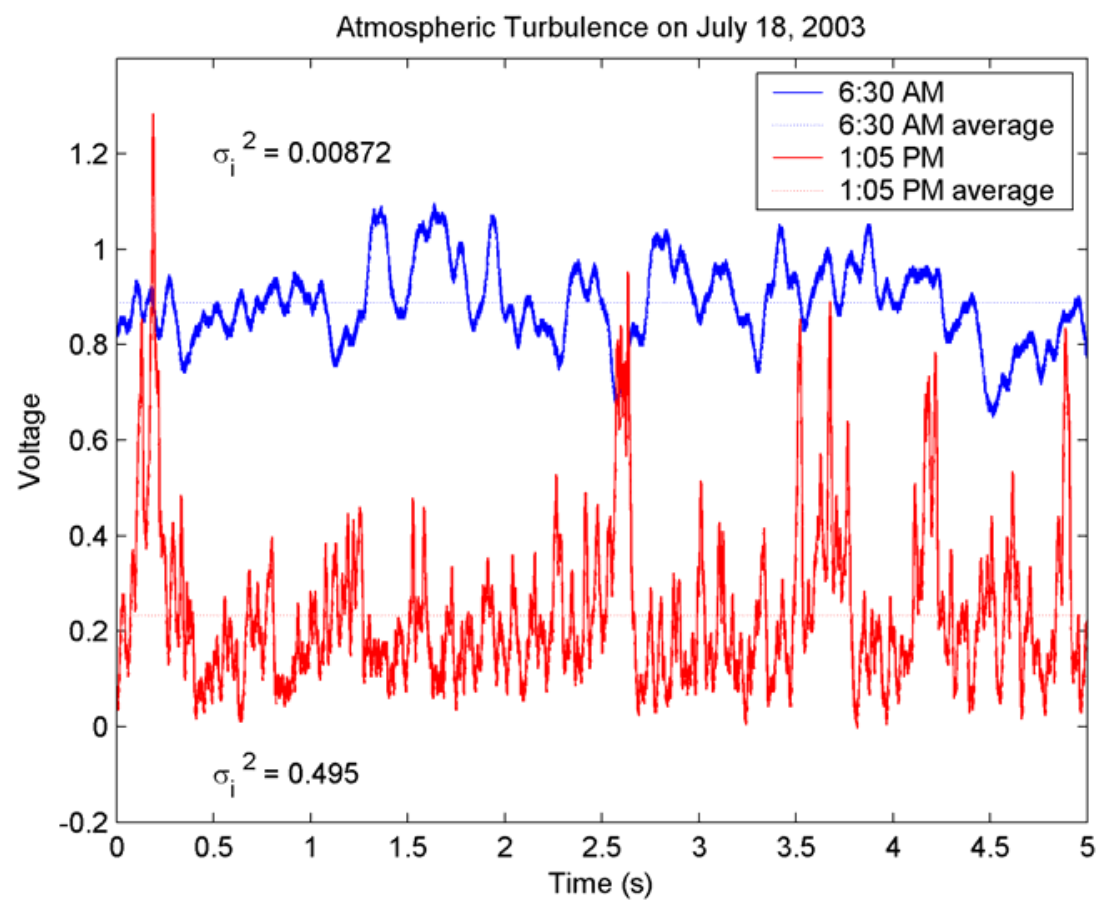

Figure 4.15. Raw Atmospheric Turbulence Data for July 18, 2003 at 6:30 AM (blue) and 1:05 PM (red). The scintillation index, $\sigma_{i}^{2}$, was 0.00872 at $6: 30$ and 0.495 at $1: 05$.

The time scale at which data becomes uncorrelated is another quantity that characterizes the atmospheric turbulence. Qualitatively, the morning data (blue) shown in Figure 4.15 exhibit smooth variation while the afternoon data (red) are choppier and more stochastic. Computation of the auto- 
correlation results in a more quantitative description. Figure 4.16 shows the computed autocorrelation for both the 6:30 AM (blue) and 1:05 PM (red) data sets. Both are completely correlated on time scales less than one millisecond and completely uncorrelated on time scales greater than half a second. That is to say, in all likelihood, two points taken 500 microseconds apart will have nearly the same value, while two points taken one second apart will be unrelated.

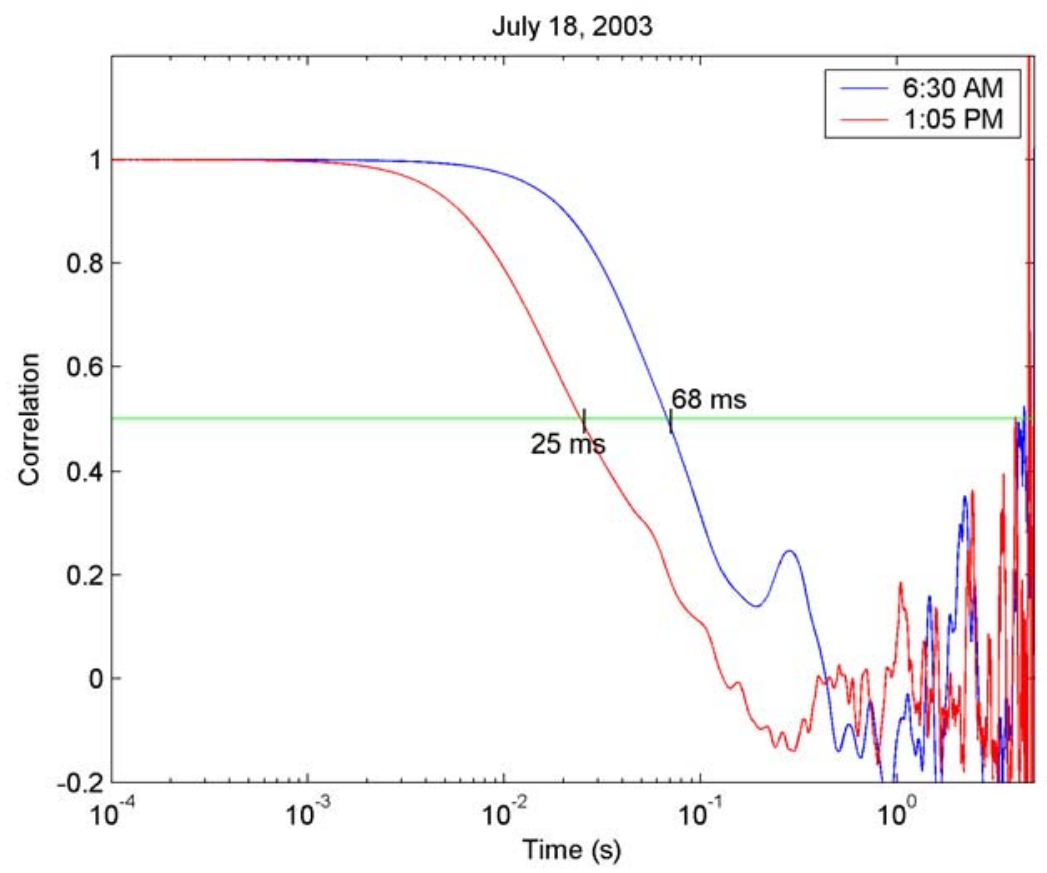

Figure 4.16. Autocorrelation Data for July 18, 2003 at 6:30 AM (blue) and 1:05 PM (red). The 6:30 data has a $50 \%$ correlation threshold of $68 \mathrm{~ms}$ while the 1:05 data has a threshold of $25 \mathrm{~ms}$.

While both data sets have the same starting and ending values, the transition between correlated and uncorrelated timescales is very different. The afternoon data set becomes uncorrelated on a faster time scale than the morning. For example, in the afternoon the data is $50 \%$ correlated after $25 \mathrm{~ms}$ while the morning data is still $50 \%$ correlated after $68 \mathrm{~ms}$ - nearly a factor of three longer-indicating the turbulence induced fluctuations are changing faster in the afternoon than in the morning. This could result from either an increased wind speed, or an increase in the convective "bubbling" of the atmosphere due to a vertical temperature gradient near the earth's surface.

Figure 4.17 shows how the scintillation index changes throughout the day. Atmospheric turbulence data was collected over a $1 \mathrm{~km}$ path length on July 17, 18, and 24, 2003. The atmosphere undergoes a surface layer stability transition approximately one hour after sunrise during which time the scintillation index is at a minimum. The atmospheric conditions gradually degrade and become more scattered as the day progresses. Recent results from Gable Mountain indicate that the scintillation index begins to decrease after midday and goes through another minimum near sunset. This indicates that dawn and dusk are the best time to perform experiments. 


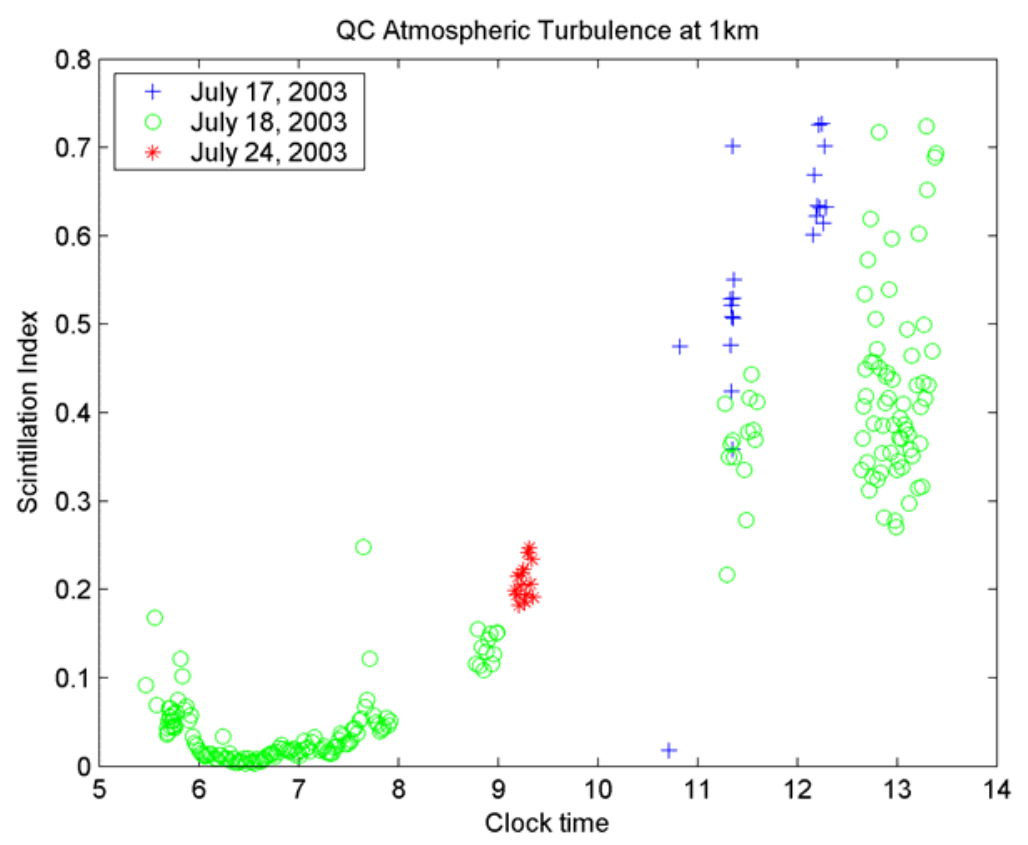

Figure 4.17. Scintillation Index from 5 AM to 2 PM on Three Different Days in July 2003. Sunrise was approximately 5:30 AM.

Figure 4.18 shows the time scale when subsequent data points are $50 \%$ correlated. Unlike the scintillation index, the data is scattered in the morning with a slightly higher average value indicating that the atmosphere is calmer than the afternoon, consistent with the scintillation index.

In studying the data shown in Figures 4.17 and 4.18, several tantalizing questions arise. Is the overall trend and scatter exhibited in the scintillation index from July 18 typical for the summer? Why is the scintillation index more clustered on July 17 than 18 ? The average correlation time scale is nearly uniform throughout the day, but there are instants when the atmosphere is nearly four times calmer in the morning, why? What were the wind speeds and directions and how would they impact the correlation data?

One of our goals for the coming year is to collect more atmospheric turbulence data in an effort to better understand the variations hour to hour, day to day and season to season. We will also use a meteorological station to measure the environmental conditions and a scintillometer to measure the index of refraction structure constant and inner scale of atmospheric turbulence- - both of which contribute to the scintillation index. This data, combined with simultaneous FM-DIAL data should help us to better understand the impact of different environmental and atmospheric conditions on our detection limit. 


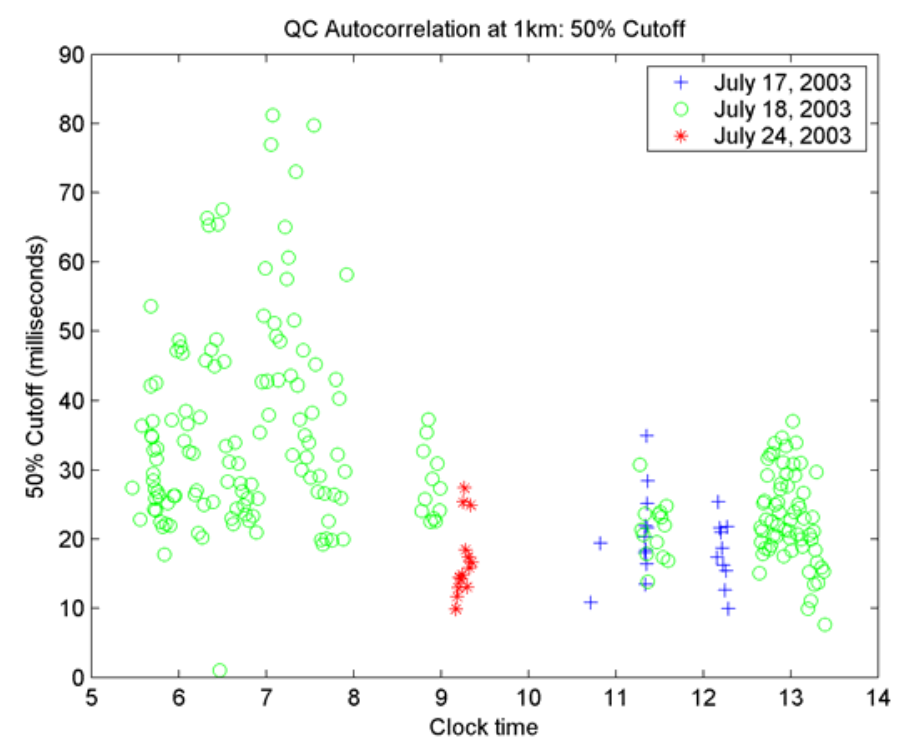

Figure 4.18. Time Scale When Subsequent Points are 50\% Correlated from 5 AM to 2 PM from Three Different Days in July

\subsection{QCL Beam Spread and Relation to Experimental Noise}

During the HTS I and HTS II field experiments in March and February, we observed that the returned laser beam was broadened at the focal point of the telescope. This problem had not been previously observed in shorter-range experiments or theoretical modeling predictions. Nominally, the telescope should focus the returned laser signal to a diffraction limited spot size with an Airy disk of about $60 \mu \mathrm{m}$ in diameter. The detector is a $1 \mathrm{~mm}$ diameter element, which is about sixteen times larger than the Airy disk. Unfortunately, one of the effects of atmospheric turbulence is to broaden imaged laser spots. Experiments are underway to determine the nature of the broadening. Two possibilities, also discussed in Section 4.4.2, are being considered: beam wander and beam broadening or beam breakup. In beam wandering, a small focal spot is maintained but it moves around within the focal plane of the telescope. In beam broadening the average position of the focal spot is constant, but the spot itself has been broadened due to refractive index gradients present in a turbulent atmosphere. We have experimentally confirmed that beam broadening does occur. As seen in Figure 4.19, spots that are 30 times larger than the diffraction limited can result.

We have also investigated the effects on the transmitted spot size of the beam at the retro-reflector. The QC laser beam was adjusted for minimum divergence over a $50 \mathrm{~m}$ path length. The resulting laser spot at $50 \mathrm{~m}$ was recorded with an infrared camera, and the image was analyzed to determine the beam parameters. The spot size was found to be $\omega_{0}=0.30 \mathrm{~cm}$, which is consistent with the output aperture of the custom optics assembly. During a recent set of field experiments, the beam was directed toward a retro-reflector at $2.4 \mathrm{~km}$ from the trailer. The returned intensity was recorded as the beam was scanned over the retro-reflector. This experiment was repeated at $5.4 \mathrm{~km}$. The resulting angular beam profiles are plotted in Figure 4.20. 


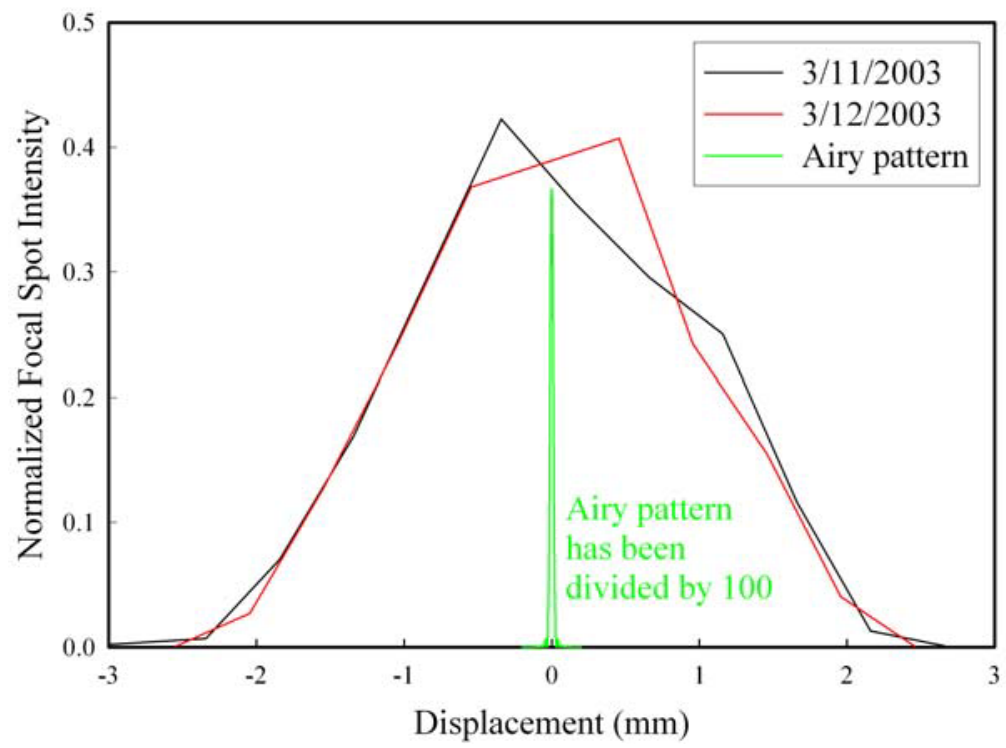

Figure 4.19. Measurement of the Focal Spot Width and Comparison with Diffraction Limited Airy Pattern. The $1 \mathrm{~mm}$ detector element was translated along one axis to determine the focused spot size. The focal spot was approximately $2 \mathrm{~mm}$ for a beam returning from a retroreflector placed at $2.3 \mathrm{~km}$.

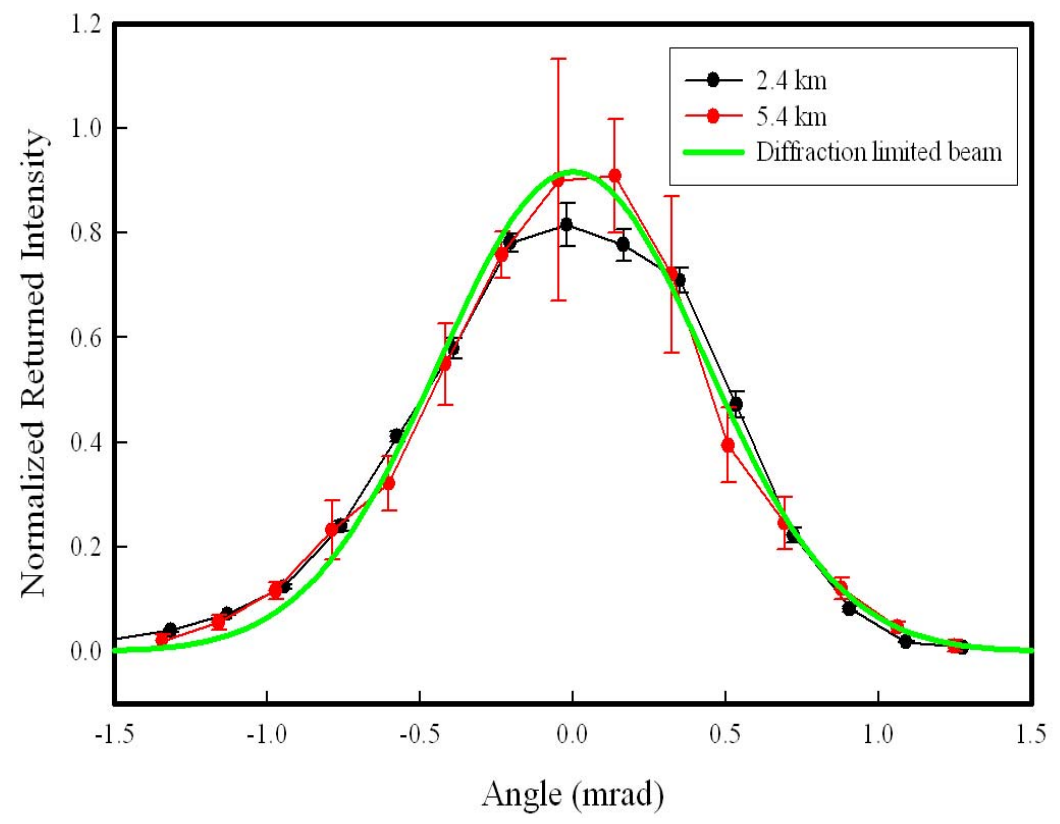

Figure 4.20. Angular Beam Profiles Taken from a Retro-Reflector Positioned at $2.4 \mathrm{~km}$ and $5.4 \mathrm{~km}$. The green trace is the expected profile assuming Gaussian beam output with $\omega_{0}=0.30 \mathrm{~cm}$. 
The profiles at $2.4 \mathrm{~km}$ and $5.4 \mathrm{~km}$ not only agree with each other, but also agree quite well with the expected output for a Gaussian beam. This suggests that transmit beam wander is not significant for these experiments. Intensity fluctuations are still present, and the mechanism is still under investigation. While the transmit beam does not seem to wander, it can still suffer beam break-up thereby causing the fluctuations.

\subsection{Impact of Large Objects Moving Through the QCL Beam}

When performing field experiments, there are a number of additional noise sources that are not normally considered in the laboratory environment. Some of these noise sources are due to objects that pass through the beam path, such as vehicles, people, deer, birds, and rain. In the March Hanford Town Site field experiments (HTS II), an unusual reduction in the received power was observed. This reduction in power occurred at about $0.4 \mathrm{~s}$ in the $34^{\text {th }}$ scan of a sequence of data, shown in Figure 4.21.

To understand the nature of this noise source, this trace was analyzed in more detail. Both the FM and RAM were observed to decrease by a proportional amount. This type of correlated reduction in the intensity is exactly what the intensity normalization procedure can handle very efficiently. After normalization, the trace is observed to have good signal to noise with no interference effects, see Figure 4.22.

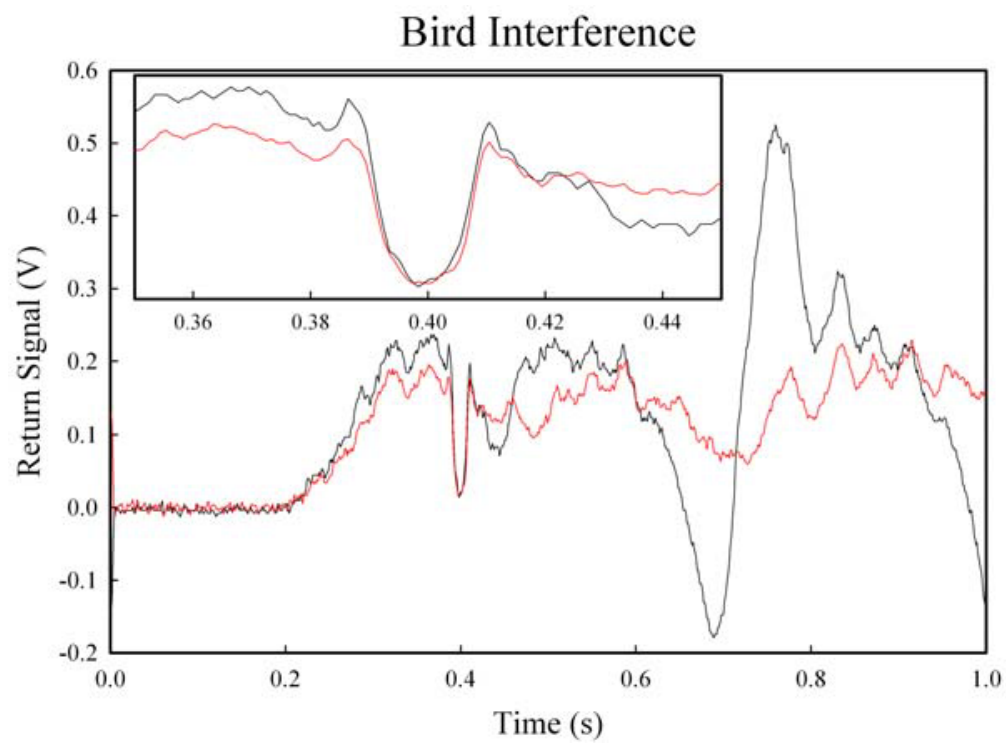

Figure 4.21. One of the Traces in a Sequence of Data Showing Reduced Intensity Returns at $0.4 \mathrm{~s}$ Due to Partial Laser Beam Blockage. The inset shows the data expanded around $0.4 \mathrm{~s}$. The black trace is the FM data and the red trace is the RAM data. 


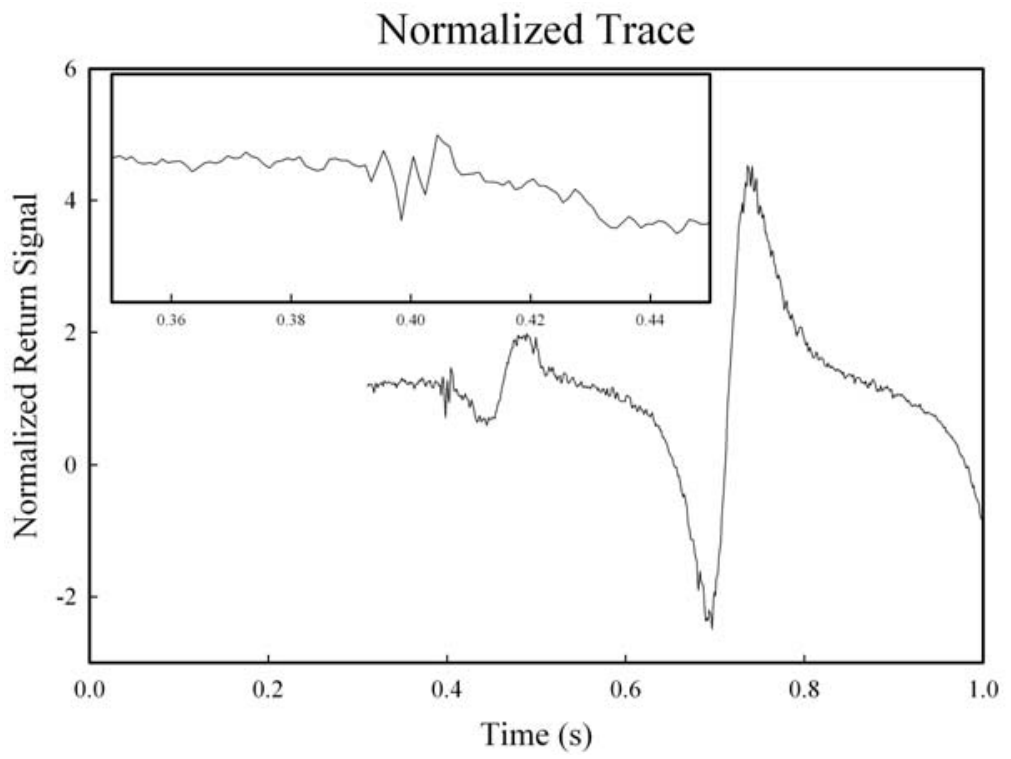

Figure 4.22. Resulting Trace After Normalizing the Data in Figure 4.21, FM Divided by RAM. The feature at $0.4 \mathrm{~s}$ is eliminated, and the result is a good trace for fitting the derivative absorption features.

Close inspection of the data in Figure 4.22 shows that while the reduction in intensity is accounted for, there is an increase in the noise level at that time. This is actually a direct consequence of the fact that the beam intensity was reduced by a factor of ten. Because the signal level dropped, and the noise in the trace stayed the same, the signal to noise ratio is degraded. The important thing to realize is that after normalization, the data is corrected for the intensity reduction but the noise of the data is increased due to lower signal to noise while the intensity is reduced.

Information about the size and speed of the object that moved through the beam can be obtained from the data in Figure 4.21 using the following analysis. Consider a beam path of width $z$, an object with width $y$, and a center displacement $x$. This simple geometry is depicted in Figure 4.23. There are three cases of interest: $y>z, y=z$, and $y<z$. The variation in returned signal for these three cases is shown in Figure 4.24.

In all cases the width at the midpoint of the transmission is equal to the object width. In cases 1 and 2 the beam is completely blocked, while in case 3 the beam is only partially blocked.

The data in Figure 4.21 clearly shows that the beam is nearly blocked, but not completely. Based on this, the conclusion is that the object is about the same size as the beam path, which is 6 " at the retroreflector. Because the data is collected in time, the speed of the object may also be measured. The duration of the obstruction (at the midpoint) is $0.02 \mathrm{~s}$. Using the derived object size (approximately $6 "$ ), and the time that it takes to move through the beam the object speed can be calculated. The speed was found to be $7.5 \mathrm{~m} / \mathrm{s}$, or $16 \mathrm{mph}$. This speed is consistent with birds observed in the area. 


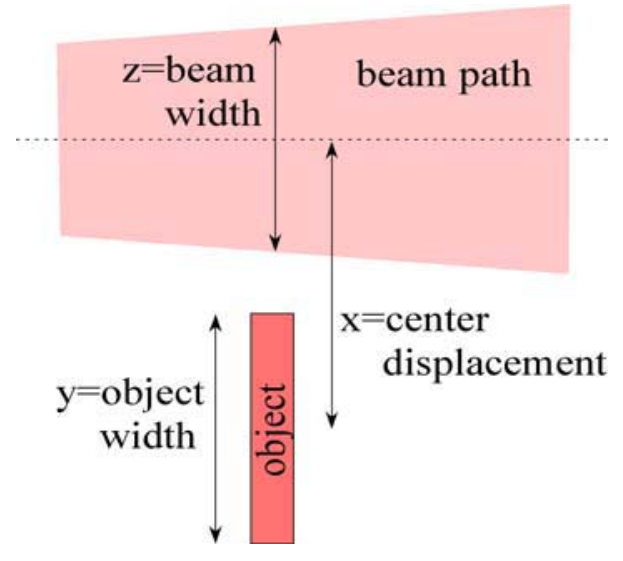

Figure 4.23. Geometry of Object Passing Through a Beam Path
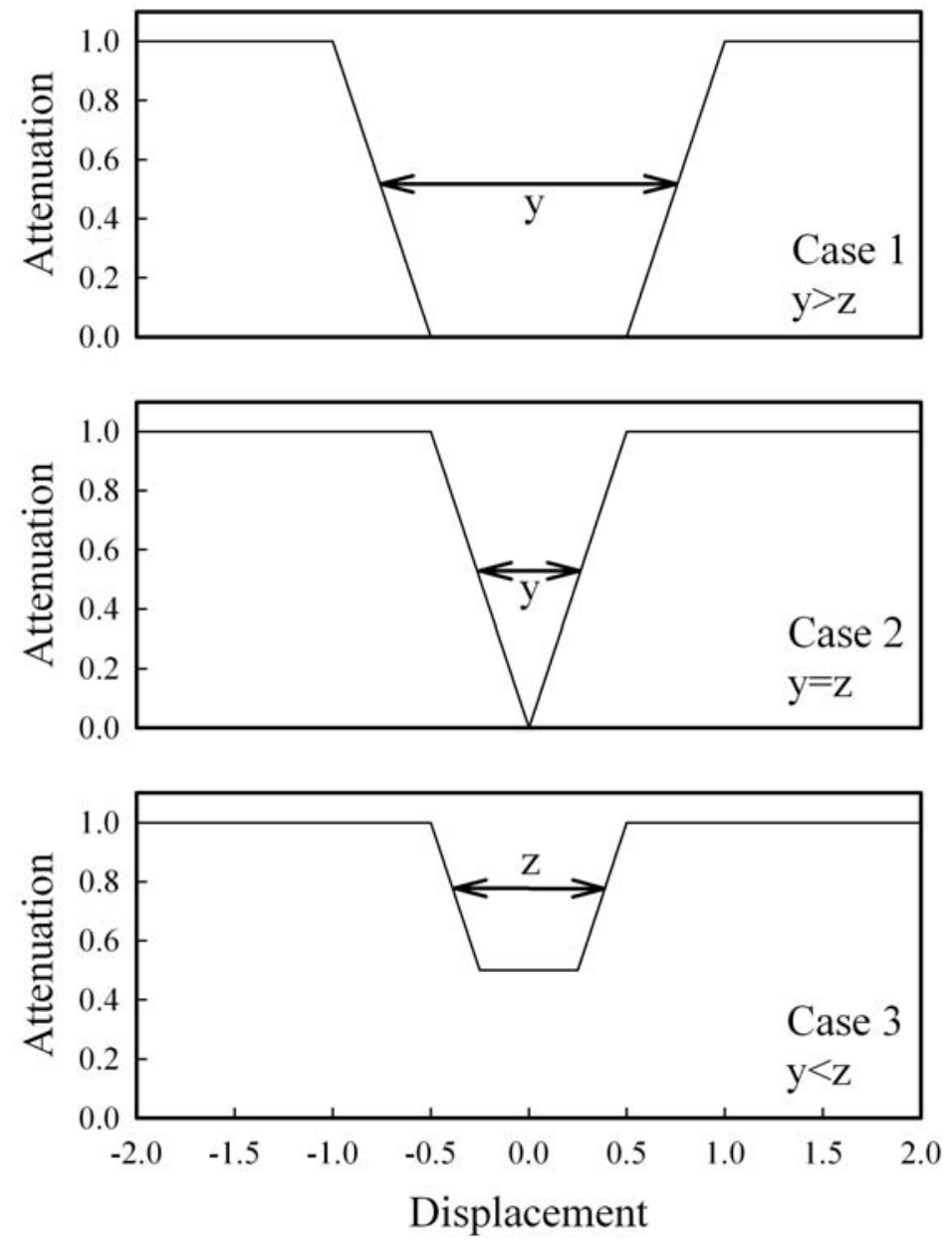

Figure 4.24. Result of Moving an Object Through a Beam Path. Each of the three cases discussed in the text are shown. 


\subsection{Real Time Data Acquisition and Analysis}

During FY03 a Labview ${ }^{\mathrm{TM}}$ software program was written to interface the digitizing electronics (a digital oscilloscope is used to digitize the lock-in amplifier analog output and other diagnostic signals) to a computer which stores and analyzes the experimental data. We currently operate in a mode where all raw data is transferred and saved to a file. Software is then used to do operator-assisted analysis on the saved data. The interface program also has the capability to continuously save data from the scope to the computer in a logging mode of operation. These features were used during the last set of field experiments. Most of the work this year relied on using the data acquisition program to do a simple preliminary analysis to ascertain the data integrity, and then directing the system to collect data in longer automated sequences.

The preliminary analysis software allows a number of quantities to be calculated and displayed. One such quantity is the autocorrelation of the returned laser intensity discussed in Section 4.4.3. The returned laser power can fluctuate as a result of the probe beam experiencing turbulence (defined here as a spatial variation in the atmospheric refractive index) along the optical pathlength. At the start of an experiment we record about a minute's worth of data on the intensity fluctuations. An autocorrelation trace is then calculated and displayed on a semi-log plot that shows the timescale for turbulence. This is illustrated in Figure 5.1. A variety options are included in this calculation so that biased, unbiased, or raw autocorrelations can be determined. The program additionally allows a variety of normalization and averaging schemes to be implemented, i.e., standard and weighted averaging (as in Figure 5.2), and normalization either before or after averaging. These features were used to perform preliminary data analysis to check the data quality before logging for more extended periods.

We are currently in the process of upgrading the data acquisition/preliminary analysis software and hardware to a more advanced state. We expect to have this completed by the end of the first FY04 quarter. Our next steps involve coding the analysis software in $\mathrm{C}^{++}$, allowing more complex functions to be added to the program without compromising the rate at which the analysis is done. The new program will interface directly with $\mathrm{A} / \mathrm{D}$ cards that are programmed to digitize the lock-in amplifier output and the experimental diagnostics that include signals from the reference cell, the reference etalon, and the laser drive current. The program will also provide true real-time data analysis, as opposed to the post-mortem evaluation we currently perform.

Several modes of operation will be available. Autocorrelation mode will allow data and analysis results to be logged continuously. Automatic processing will provide $80 \%, 50 \%$, and $20 \%$ de-correlation times that relate to the timescale of turbulence. The scintillation index will be calculated directly from the data to allow convenient comparison to theoretical modeling work. The same data traces can also be analyzed to determine signal statistics, including intensity distribution data, mean, variance, skewness, kurtosis. A second mode will allow laser wavelength scans to be recorded, averaged, normalized, and then fit to determine the absorbance and concentration path length for particular molecules. 


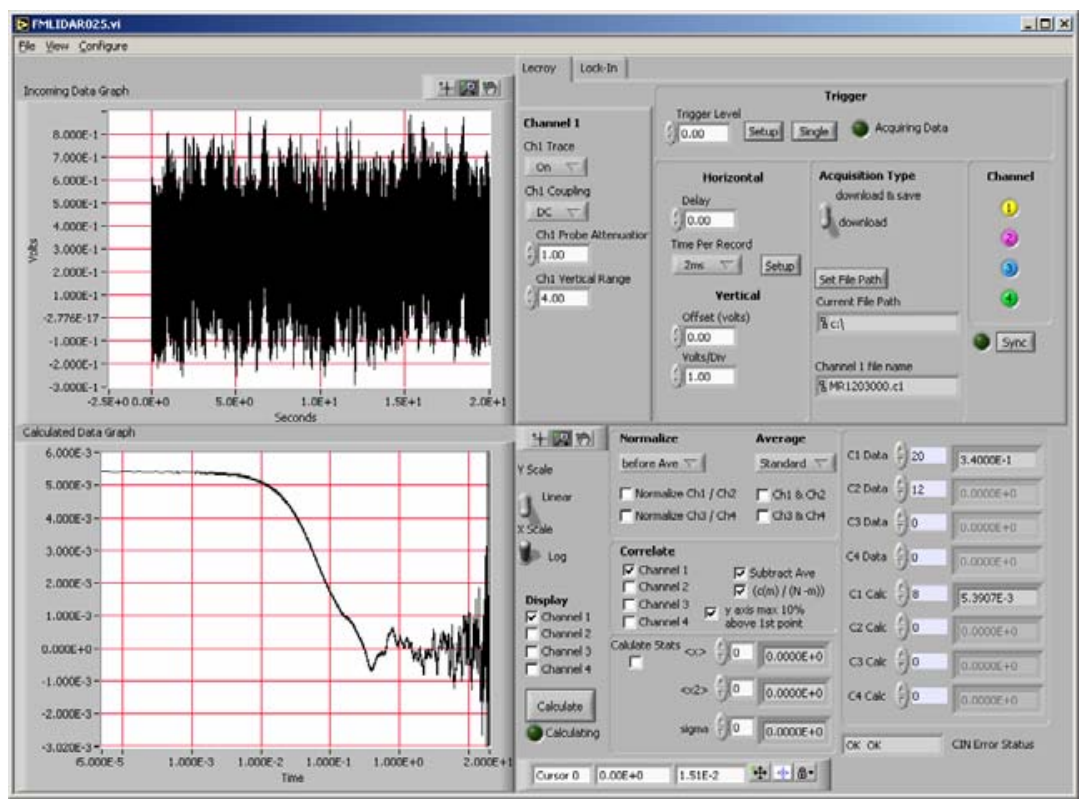

Figure 5.1. Autocorrelation Calculation. Top left is the raw data, taken over a 20 second time interval, which consists of intensity fluctuations caused by atmospheric turbulence. The bottom left panel shows the autocorrelation which indicates that there is a $50 \%$ de-correlation at just under $0.1 \mathrm{sec}$. This result shows that spectral scans should be taken in $10 \mathrm{~ms}$ or less to minimize turbulence noise.

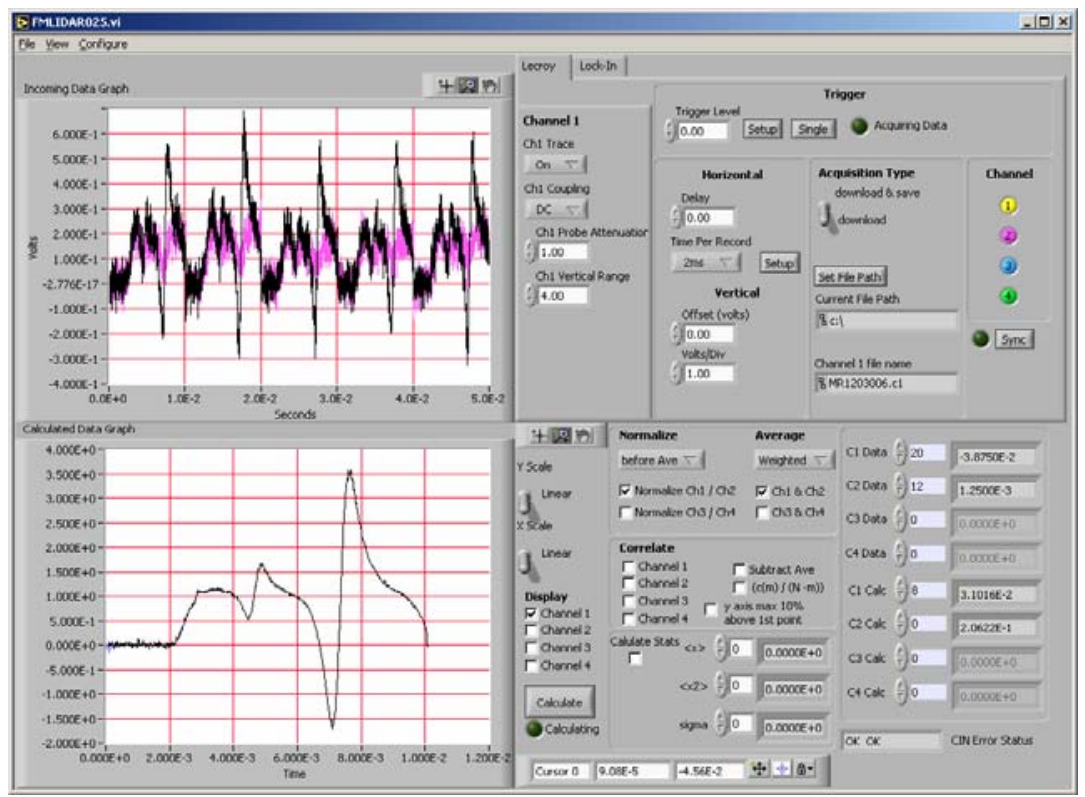

Figure 5.2. Average Calculation. The top left panel has been expanded to show the first five traces of a sequence of 512 successive scans. The bottom trace shows the result of normalizing the FM to the RAM, and calculating the weighted average of 512 traces. 
To implement real time concentration analysis into the FM DIAL experiment it is necessary that the derivative absorbance features outlined and discussed in Section 1.1 be fitted to a nonlinear least squares function. During FY03 we investigated a number of approaches for doing this. While nonlinear fitting can be a fairly slow process because of the iterations required, calculation speed can be greatly enhanced by choosing the fitting function form that best models the expected experimental results. Typically in nonlinear fitting, an initial guess is given to start the iterative calculation. In most efficient nonlinear fitting routines, each step requires calculating the derivative with respect to each fitted parameter. These derivatives, along with the observed and calculated profiles, are used to change the parameters to values that cause the calculated function to fit the data better. Nonlinear fitting techniques are well known, and the details will not be repeated here; however, it is important to realize that one of the slow steps can be calculating the Jacobian matrix (the derivatives). Essentially an $n \times m$ matrix must be created with derivatives for each parameter $(m)$ at each data point $(n)$. This calculation must also be performed at each step of the iterative process. One of the simplest ways to arrive at the derivatives is to evaluate them numerically. Each derivative is calculated from two function evaluations and in many cases this can be computationally expensive and time consuming. Another method is to use an analytic expression for the derivative lineshape formula.

Because we perform the FM DIAL experiments at atmospheric pressure it is not unrealistic to assume that the observed lineshapes will be Lorentzian in character, i.e.,

$$
g\left(v-v_{0}\right)=\frac{\left(\frac{\Delta v}{2 \pi}\right)}{\left(\frac{\Delta v}{2}\right)^{2}+\left(v-v_{0}\right)^{2}} .
$$

Here $\Delta v$ is the full-width at half-maximum (FWHM) of the peak, $v_{0}$ is the center frequency (wavelength), and $v$ is the frequency (wavelength). This functional form is typically used because the integral over all frequencies is unity. The observed signal in an FM DIAL experiment is a derivative of the lineshape, i.e.,

$$
\frac{d g\left(v-v_{0}\right)}{d v}=\frac{-2\left(v-v_{0}\right)\left(\frac{\Delta v}{2 \pi}\right)}{\left[\left(\frac{\Delta v}{2}\right)^{2}+\left(v-v_{0}\right)^{2}\right]^{2}}
$$

When performing least squares fitting for our analysis, it is useful to divide by the peak height and scale the function by an amplitude parameter, A; to obtain the unitless quantity

$$
h\left(v-v_{0}\right)=\frac{-A\left(v-v_{0}\right) \Delta v^{3}}{\sqrt{27}\left[\left(\frac{\Delta v}{2}\right)^{2}+\left(v-v_{0}\right)^{2}\right]^{2}} .
$$


The function above has been modified to make $A$ the peak-to-peak amplitude of the derivative lineshape. This quantity is directly incorporated into our algorithms for calculating the observed absorbance and concentration path length product. In efforts to increase the speed of nonlinear least squares fitting, we have derived the following analytical expressions for the derivatives with respect to each of the parameters;

$$
\begin{aligned}
\frac{d h\left(v-v_{0}\right)}{d v}= & \frac{-A \Delta v^{3}}{\sqrt{27}\left[\left(\frac{\Delta v}{2}\right)^{2}+\left(v-v_{0}\right)^{2}\right]^{2}}+\frac{4 A\left(v-v_{0}\right)^{2} \Delta v^{3}}{\sqrt{27}\left[\left(\frac{\Delta v}{2}\right)^{2}+\left(v-v_{0}\right)^{2}\right]^{3}}, \\
\frac{d h\left(v-v_{0}\right)}{d v_{0}}= & \frac{-A \Delta v^{3}}{\sqrt{27}\left[\left(\frac{\Delta v}{2}\right)^{2}+\left(v-v_{0}\right)^{2}\right]^{2}}-\frac{4 A\left(v-v_{0}\right)^{2} \Delta v^{3}}{\sqrt{27}\left[\left(\frac{\Delta v}{2}\right)^{2}+\left(v-v_{0}\right)^{2}\right]^{3}}, \\
\frac{d h\left(v-v_{0}\right)}{d \Delta v}= & \frac{-A\left(v-v_{0}\right) \Delta v^{2}}{\sqrt{3}\left[\left(\frac{\Delta v}{2}\right)^{2}+\left(v-v_{0}\right)^{2}\right]^{2}}-\frac{A\left(v-v_{0}\right) \Delta v^{4}}{\sqrt{27}\left[\left(\frac{\Delta v}{2}\right)^{2}+\left(v-v_{0}\right)^{2}\right]^{3}}, \\
& \frac{d h\left(v-v_{0}\right)}{d A}=\frac{-\left(v-v_{0}\right) \Delta v^{3}}{\sqrt{27}\left[\left(\frac{\Delta v}{2}\right)^{2}+\left(v-v_{0}\right)^{2}\right]^{2}} .
\end{aligned}
$$

Use of these analytic expressions allows direct calculation of the derivatives without multiple evaluations of the derivative line shape formula. Use of the analytic formulae will provide more accurate derivatives for the fitting procedures, and should be faster than the calculation of numeric derivatives. 


\subsection{FM DIAL Detection Limit}

Our currently accepted definition of the experimental detection limit is the minimum single result which can be distinguished from a suitable blank value, with a $1 \sigma(68 \%)$ confidence level. For absorption experiments, we directly measure the observed absorbance, so the best quantity to report is the absorbance detection limit (unitless). Often, there is more interest in how well the experiment can detect a particular chemical. In those cases, use of an absorption cross section can be used to give the detection limit in terms of a chemical concentration. There are many possibilities for the units of this type of detection limit, but the most common are $\mathrm{ppm}_{\mathrm{v}}, \mathrm{ppm}_{\mathrm{v}} \times \mathrm{m}, \mathrm{kg} / \mathrm{m}^{3}$, torr, or torr $\times \mathrm{m}$. The units are typically a concentration, or a concentration $\times$ distance. For path-integrated experiments, such as FM DIAL, the concentration $\times$ distance is probably the best choice. This unit quantifies the amount of chemical that can be detected using a $1 \mathrm{~m}$ path length. For plume release experiments it is important to remember that it is the path through the chemical plume, and not the distance to the retro-reflector, which determines the distance scale associated with concentration $\times$ distance detection limits.

We have determined the detection limit for several situations in the past year. It is important to realize that the detection limit changes as a function of many variables including distance to the retro-reflector, laser power, humidity, detection geometry, turbulence conditions, chemical of interest, and many others. The main experiments that we have performed include the Hanford Town Site (HTS) tests, the Meteorological tower tests, and the Gable Mountain tests. The HTS tests targeted ambient water vapor as the target chemical. Numerous tests were performed looking at the detection limit as a function of laser ramp frequency and distance to the retro-reflector. The Met tower tests were a collaborative effort in which hydrogen sulfide $\left(\mathrm{H}_{2} \mathrm{~S}\right)$ was released in an air plume. The goal of these experiments was to observe small quantities of $\mathrm{H}_{2} \mathrm{~S}$ and determine the detection limit. The Gable Mountain experiments used a flip-in cell filled with a small quantity of nitrous oxide. Tests were performed with, and without, the gas cell in the beam and as a function of distance to the retro-reflector, and time of day.

One important consideration in FM DIAL returns is the detection limit dependence on the distance to the retro-reflector. During the HTS tests we probed this dependence, and recent results from Gable Mountain will give explicit data on the distance dependence. A set of data from the HTS experiments looked at two distances, $2.32 \mathrm{~km}$ and $4.70 \mathrm{~km}$. Experimental data was collected at each of the two ranges within a short period of time such that turbulence and other effects were relatively unchanged. The column integrated water concentration results agreed well with each other, after scaling by 2.03 to compensate for the longer path length to the $4.7 \mathrm{~km}$ range. After analysis of the data, the detection limit for the $2.32 \mathrm{~km}$ range was 8.6 times better than that seen for the $4.70 \mathrm{~km}$ range. This is consistent with the expected decrease in return power levels, which at these ranges should be between $\mathrm{R}^{2}$ and $\mathrm{R}^{4}$ as diffraction from the retro-reflector aperture starts to contribute. In these experiments the observed absorbance detection limit was $1.6 \times 10^{-4}$ for 5 seconds of averaging at $2.32 \mathrm{~km}$, without resorting to chemometrics analysis methods. Fitting an actual data series and analyzing the resulting statistical fluctuations obtained the detection limit. The actual concentration detection limit for water at the $2.32 \mathrm{~km}$ range was $2.4 \mathrm{ppm}$ in 5 seconds. Application of additional chemometrics methods is expected to lower the detection limit significantly, as the case for the hydrogen

sulfide release experiments. The detection limit for the $4.7 \mathrm{~km}$ range was about $1.4 \times 10^{-3}$ for 5 seconds of averaging. Other water transitions have much more favorable absorption cross-sections, up to 5 orders of 
magnitude larger. This would suggest detection limits of 13 parts per trillion $(60 \mathrm{ppb} \times \mathrm{m})$, however this is not possible given the amount of water in ambient air - one of these stronger lines would completely absorb the transmitted laser beam after only several meters. There could be applications for detection of isotopes of water, including $\mathrm{HDO}, \mathrm{D}_{2} \mathrm{O}, \mathrm{HTO}, \mathrm{DTO}$, and $\mathrm{T}_{2} \mathrm{O}$. These isotopes have similar absorption cross-sections, and should be detectable at parts per trillion levels with suitable pathlengths.

The Met tower experiments (hydrogen sulfide release campaign) provided additional information about the detection limit for a practical application. In these experiments, small quantities of hydrogen sulfide $\left(\mathrm{H}_{2} \mathrm{~S}\right)$ were released in the form of a plume. Data was collected using a retro-reflector positioned $155 \mathrm{~m}$ from the trailer. $\mathrm{H}_{2} \mathrm{~S}$ concentrations up to $500 \mathrm{ppm}$ were released in a plume configuration, which quickly diluted when mixed with air. During the data analysis, an extended least squares chemometrics method was used. This involved collecting background variability data that was used to construct a 'training set'. The training set was then used in the data fitting procedure to reduce and eliminate background noise sources. The results of using this procedure was an absorption detection limit of $1.5 \times 10^{-5}$, and a concentration detection limit of $1.8 \mathrm{ppm} \times \mathrm{m}$. The $\mathrm{H}_{2} \mathrm{~S}$ detection limit could be lowered to $425 \mathrm{ppb} \times \mathrm{m}$ by using a QCL wavelength optimized to a stronger transition. Application of more advanced chemometric methods could also lower the detection limit.

Until recently, all experiments were performed with either ambient water vapor or a chemical release plume. For detection limit measurements these scenarios have some shortcomings. One of the most serious problems is changes in the target chemical concentration. For ambient water vapor, the concentration can fluctuate as the wind moves different air pockets through the detection volume. These fluctuations tend to decrease over longer integrated path lengths, but can still be significant. Another problem with ambient water vapor measurements is that water is detected with no interference from other molecules, which can distort the detection limit. When chemicals are released as a plume, there are significant fluctuations in the columnintegrated concentrations along the beam path. These fluctuations are due to the wind effects and mixing effects as the released chemical dilutes into the air.

More recently, we have started to use a better method for determining the detection limit that overcomes the above mentioned problems. We have modified the FM DIAL optics train to allow a sample cell to be included in the beam path (just before the laser is directed out to the retro-reflector). The gas in the sample cell can be set to have an appropriate amount of chemical for detection limit experiments. The chemical is buffered up to one atmosphere so that it is equivalent to observing trace chemicals in the atmosphere. Because we are performing path-integrated measurements, our experiment is not sensitive to how the target chemical is distributed over the path of the laser as it goes to (and from) the retro-reflector. This technique allows observation of the absorption features of a chemical that has no variations in concentration. After applying intensity normalization techniques and fitting procedures to calculate the absorbance (or concentration), any fluctuations observed can then be assigned to instrument noise sources rather than target chemical concentration fluctuations. This method also affords us the opportunity to gauge how reproducible our concentration estimates are over time. We can also perform experiments in this way with chemicals that are undesirable to release in quantities necessary to generate a plume for detection. We have already started to collect data from these types of experiments. During the Gable Mountain experiments we used a cell with nitrous oxide that could be added and removed from the beam path. Analysis is still in progress, but the data will allow testing of the fitting procedures and development of more powerful chemometrics methods. 


\subsection{Chemical Release Experiments}

This July we performed a field campaign aimed at detection of small quantities of hydrogen sulfide, $\mathrm{H}_{2} \mathrm{~S}$. These tests were conducted near the meteorological tower near the center of the Hanford site. The trailer was setup at the intersection of two roads, so that several beam paths would be available to match the prevailing wind direction.

A 'skid' apparatus was borrowed from the PNNL's Hydrology and Chemistry group. Originally, the apparatus was used to pump $\mathrm{H}_{2} \mathrm{~S}$ into groundwater monitoring wells for experiments aimed at reducing soil contamination. The apparatus, shown in Figures 7.1 and 7.2, was capable of forcing air through a 3" PVC pipe at flow rates in excess of $100 \mathrm{cfm}$ to form the plume. Gas rotometers allowed the addition of small quantities of $\mathrm{H}_{2} \mathrm{~S}$ to the airflow, so that the $\mathrm{H}_{2} \mathrm{~S}$ concentration at the start of the plume was $<500 \mathrm{ppm}$. Controls allowed the amount of $\mathrm{H}_{2} \mathrm{~S}$ to be regulated or turned off so that experiments could be performed in the absence of $\mathrm{H}_{2} \mathrm{~S}$, without turning off the airflow.

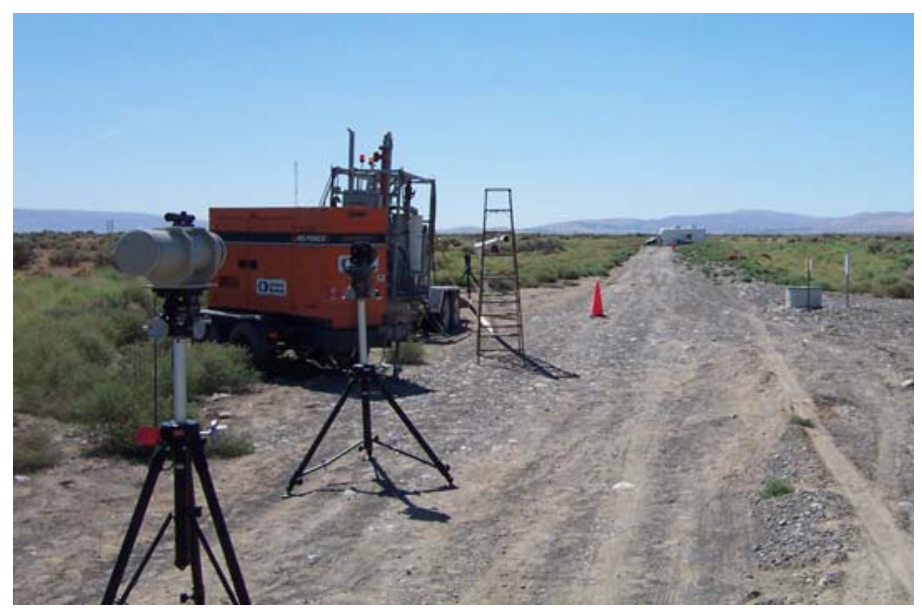

Figure 7.1. View from the Retro-Reflector Toward the Trailer

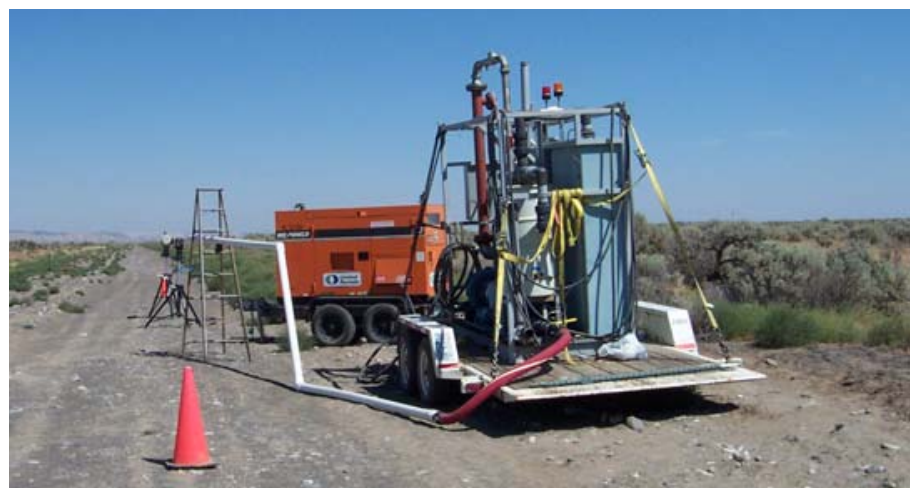

Figure 7.2. $\mathrm{H}_{2} \mathrm{~S}$ Release Skid. The retro-reflector and heated sender unit can be seen just to the left of the ladder. 
A 6" retro-reflector was placed about $155 \mathrm{~m}$ from the trailer to provide good laser return signals for the experiment. Because a small plume (3" at start) was used, it can be difficult to maintain the plume in the detection region. The output of the skid was positioned 7 yards before the retro-reflector, and the plume was directed to cross the beam path in front of the retro. This arrangement helps ensure that the plume will remain in the detection region, even when the prevailing winds shift. While the release concentration was $500 \mathrm{ppm}$, the observed concentration is expected to be significantly smaller. As the gas exits the 3" tube, it quickly starts to mix and dilute with air. As the plume continues to expand, the concentration of $\mathrm{H}_{2} \mathrm{~S}$ is expected to decrease with the square of the distance from the release point. The beam path was horizontal, remaining within $2 \mathrm{~m}$ above ground height.

The FM DIAL system used both an $8.2 \mu \mathrm{m}$ quantum cascade laser and a $1.56 \mu \mathrm{m}$ commercial laser system. The QCL was not specifically optimized for $\mathrm{H}_{2} \mathrm{~S}$ detection but could be used because several transitions occur within the laser's extended tuning range. The transition probed was nearly five times weaker than other $\mathrm{H}_{2} \mathrm{~S}$ absorption features that could be accessed with an optimized system. The experiment was arranged so that the operator could manually switch between the two laser wavelengths.

In addition to the FM DIAL system, our FTIR sensor was deployed in the active configuration using a heated sender unit. The sender telescope was positioned just behind and to the side of the retro-reflector such that the beam path was nearly identical to the FM DIAL beam path. The collimated sender beam was received by a telescope and directed into the Bruker FTIR spectrometer. The FTIR spectra were recorded simultaneously with the FM DIAL experiments. Figure 7.3 shows the FM DIAL and FTIR systems.

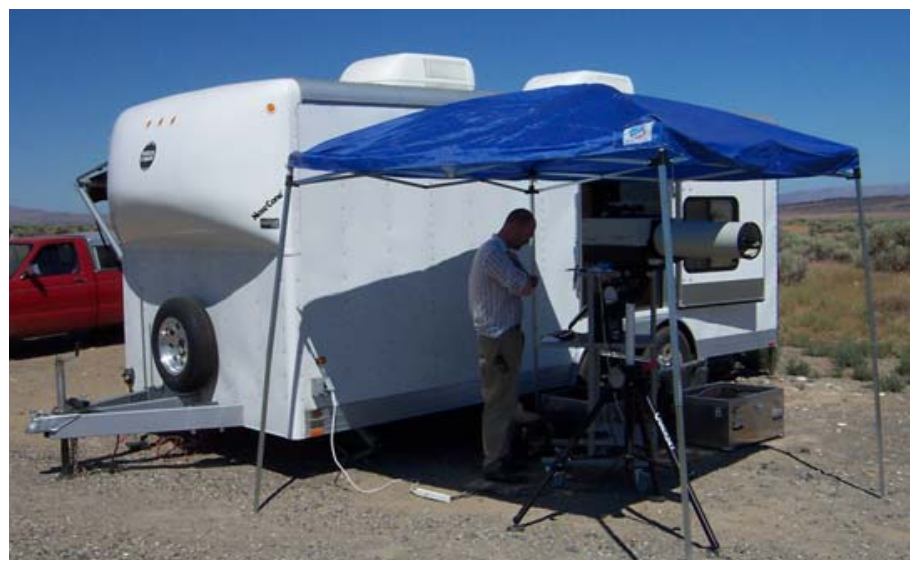

Figure 7.3. FM DIAL Trailer and the FTIR Sensor System. Beam paths were aligned such that they crossed close the chemical release point.

QCL FM DIAL data collected from the hydrogen sulfide chemical release experiments showed small absorption signals due to $\mathrm{H}_{2} \mathrm{~S}$ and larger signals from ambient water features. The data clearly show $\mathrm{H}_{2} \mathrm{~S}$ absorption features, and the trained eye could see these in individual data traces. The data in Figure 7.4 shows the absorption features after eliminating the effects due to ambient water absorption and baseline fluctuations. 


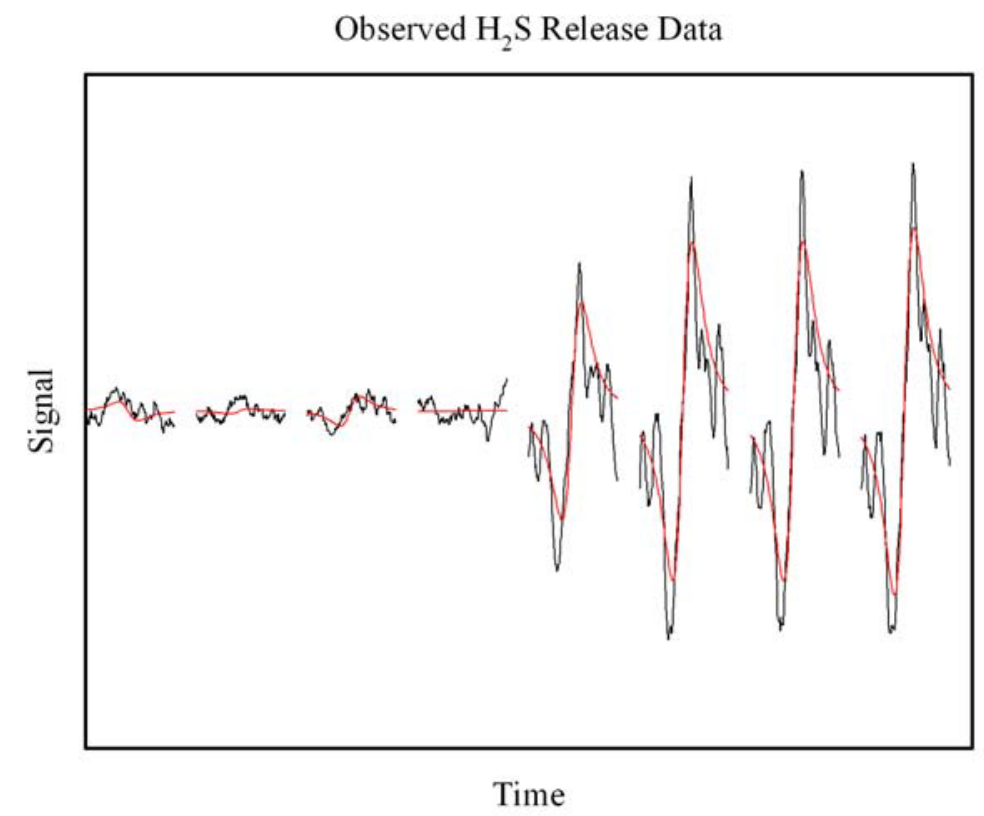

Figure 7.4. Selected Averaged Waveforms Showing the Derivative Absorption Features Due to $\mathrm{H}_{2} \mathrm{~S}$. On the left side is the data with no gas released, and on the right side are the features observed at different times after the release was initiated (QCL data).

We performed an analysis of the data at several levels. The simplest approach was to fit only the $\mathrm{H}_{2} \mathrm{~S}$ absorption feature and surrounding baseline. This simple analysis was easily able to observe an incremental change when the $\mathrm{H}_{2} \mathrm{~S}$ was released; however, there were some problems due to interference with weak water lines and experimental fluctuations. This interference caused offsets and some instability in the concentration estimation. We also pursued more advanced analysis techniques including extended least squares fitting. This procedure makes use of the data when the $\mathrm{H}_{2} \mathrm{~S}$ is known to be absent, and accumulates a 'training set'. The training set can be used to eliminate noise sources that are known not to be due to the target chemical. A more detailed treatment using extending least squares is still in progress, but the preliminary results are very encouraging. Figure 7.5 shows the results of extended least squares treatment, and the resulting concentration estimation has a number of good properties. First, the predicted absorbance is centered on zero when no signal is present. Secondly, there is no observed drift in the baseline. The data clearly shows the change in the observed signal as the $\mathrm{H}_{2} \mathrm{~S}$ is released. In fact, the small bump just before waveform 50 is real and corresponds to the operator turning on the chemical release. Each point is an average of five seconds of data. Using the absorbance estimation algorithm, the data presented here corresponds to an absorbance of $1 \times 10^{-3}$. Using the known cross-section for this $\mathrm{H}_{2} \mathrm{~S}$ transition, the absorbance corresponds to a $125 \mathrm{ppm} \times \mathrm{m}$ concentration. This concentration is consistent with what is expected for the region of the plume interrogated for the FM DIAL experiments. From analysis of the off signal data in Figure 7.5, the signal to noise of the observed signals was approximately 70 (for the extended least squares estimates). Therefore the realized detections sensitivity was $1.5 \times 10^{-5}$, or $1.8 \mathrm{ppm} \times \mathrm{m}$ for 5 seconds of averaging for these experiments at a range of $150 \mathrm{~m}$. Even using the same beam path, simultaneous FTIR experiments were unable to detect $\mathrm{H}_{2} \mathrm{~S}$ release at these levels. 


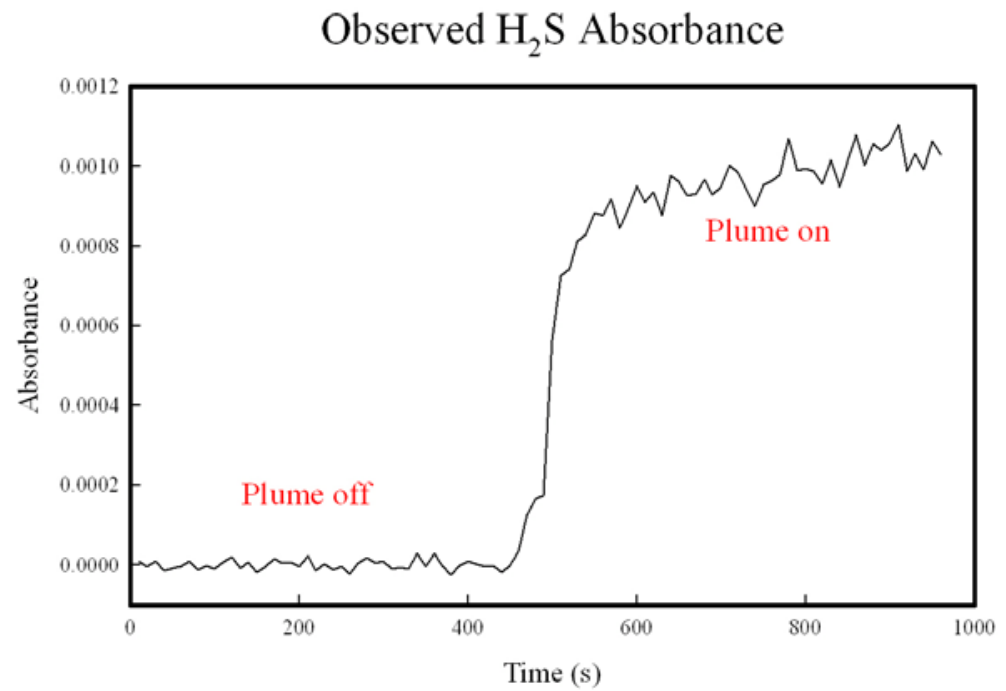

Figure 7.5. Results from the Extended Lease Squares Treatment 


\subsection{FY04 Research Plans}

The proposed plan for the FM DIAL project at PNNL in FY04 includes the following areas of emphasis.

- Order a QCL optimized for a relevant chemical, implement this laser into system, perform release experiments, and measure the detection limit.

- Develop the empirical correlation between FM DIAL performance and atmospheric turbulence.

- Perform additional field experiments monitoring $\mathrm{H}_{2} \mathrm{~S}, \mathrm{~N}_{2} 0$, Freon-134a, the relevant chemical identified in Section 8.1 and HF (if release experiments planned by NA-24 proceed).

- Determine feasibility of pulsed broadband FM DIAL.

- Investigate the impact that FM DIAL collection geometry has on performance.

\subsection{Optimized QCL}

The FM DIAL experiment is currently using an $8.2 \mu \mathrm{m}$ QCL because this was the only device available at the time the instrument was assembled. While we have been able to monitor $\mathrm{H}_{2} \mathrm{O}, \mathrm{N}_{2} \mathrm{O}, \mathrm{CH}_{4}$, $\mathrm{H}_{2} \mathrm{~S}$ and Freon-134a with this laser, its wavelength does not coincide with any of these species strongest absorption lines. Because the detection limit is optimized when the absorption cross section of the optical transition being probed is at its maximum, it would be advantangeous to have a laser whose wavelength is tailored for the particular analyte species being targeted. In FY04 we are going to identify a species pertinent to WMD proliferation and amenable to FM DIAL probing (i.e., the species transition linewidth must be smaller than the QCL tuning range), determine the wavelength of maximum absorption (via either a literature search or in cooperation with PNNL's spectroscopy group), and order a QCL that lases at that wavelength.

\subsection{FM DIAL Performance and Atmospheric Turbulence}

To develop a better FM DIAL data analysis algorithm, we need to quantify the relation (or at least develop an empirical correlation) between local turbulence, FM DIAL sensor noise and intensity fluctuations, and atmospheric background variability. We will therefore conduct three multi-instrument, multi-measurement field campaigns during FY04. The experiments will be conducted at or around the Hanford 200 site area and will be done in the fall, winter, and spring (we already have data for the summer). Scintillometer, meteorological, and FM DIAL sensor measurements (in both the LWIR and SWIR) will be collected in parallel to provide spatially and temporally correlated data. The data collected from the three instruments will be mined to determine if any correlations exist, and if so, the correlation will be quantified. In addition, a comparison of the SWIR and LWIR FM DIAL sensor performances will allow us to determine the feasibility of incorporating the broadband external cavity quantum cascade lasers into the FM DIAL system. 


\subsection{Additional Field Experiments}

In FY03 we initiated a series of $\mathrm{H}_{2} \mathrm{~S}$ release experiments in conjunction with PNNL's Hydrology and Chemistry group. We plan to continue these experiments during FY04. Specifically, we are planning releases in the fall, winter and spring, so as to investigate the influence that variations in the external ambient environment have on FM DIAL performance. In addition, a $\mathrm{UF}_{6}$ release is currently being planned for FY04. We have volunteered to be a part of this mission and will use the FM DIAL, outfitted with the appropriate SWIR laser, to detect HF, a product of $\mathrm{UF}_{6}$ hydrolysis. Where and whenever possible we are also planning to continue release studies using $\mathrm{N}_{2} \mathrm{O}$ and Freon-134a. Since these are nontoxic and non controlled substances it will be possible to perform release experiments in a variety of venues.

\subsection{Pulsed Broadband FM DIAL Feasibility}

One key shortcoming of our current QC laser-based FM DIAL system is that it can be used to monitor only a small subset of gas phase chemical species. The reason for this is that current QC lasers can be scanned over only about $1.5 \mathrm{~cm}^{-1}$. Most of the chemical species associated with CBN weapons proliferation have absorption lines that are on the order of 20 to $50 \mathrm{~cm}^{-1}$ wide. For unambiguous FM DIAL detection, the molecular absorption must be scanned over at least one side of the absorption peak. Hence the laser used in the experiment must be capable of being scanned over 10 to $25 \mathrm{~cm}^{-1}$. Gmachl (Lucent and Princeton University) has recently demonstrated broadband QCL emission over $\sim 100 \mathrm{~cm}^{-1}$. In FY04 we will try to get one of these early generation lasers, incorporate it into the FM DIAL experiment, and conduct a "large molecular species" release experiment. The particular species that will be used depends upon the new QCL emission profile. In the event that a broadband QCL cannot be acquired we will investigate an alternate detection scheme which involves incorporating a broadly tunable (3-4 $\mu \mathrm{m})$ LWIR optical parametric oscillator (OPO) into the system. Experiments using this pulsed laser system will have to be conducted using a time-gated detection scheme. Hereafter we will call this technique Pulsed DIAL. The means to implement this will be identified in FY04 and the specifications for a custom built LWIR OPO system will be negotiated with Acculite who may be commissioned to build the system for delivery in early FY05.

\subsection{FM DIAL Collection Geometry}

Thus far our FM DIAL field tests have principally been done using a "horizontal" geometry, i.e., the laser is propagated parallel to the ground. Since it is possible that some monitoring scenarios may preclude using this type of geometry it is important that we investigate and quantify the impact that different collection geometries (horizontal upward, downward, and vertical paths) have on FM DIAL performance and detection limit, and finding correlations between detection limit and atmospheric conditions. 


\section{Distribution}

No. of

\section{Copies}

\section{OFFSITE}

Mr. W. Randy Bell

United States DOE

NNSA/NA-22

1000 Independence Ave. SW

Washington, DC 20585

Dr. David Berry

United States DOE

NNSA/NA-22

1000 Independence Ave. SW

Washington, DC 20585

LTC John C. Carrano, PhD

Program Manager

DARPA, MTO

3701 N. Fairfax Dr.

Arlington, VA 22203-1714

Mr. Ralph Hastings

United States DOE

NNSA/NA-22

1000 Independence Ave. SW

Washington, DC 20585

Mr. Eric Sanders

United States DOE

NNSA/NA-22

1000 Independence Ave. SW

Washington, DC 20585
No. of

Copies

Dr. Vaughn Standley

United States DOE

NNSA/NA-22

1000 Independence Ave. SW

Washington, DC 20585

\section{ONSITE}

\section{Pacific Northwest National Laboratory}

$\begin{array}{ll}\text { Aker, PM } & \text { K5-25 } \\ \text { Cannon, BD } & \text { K5-25 } \\ \text { Doherty, TJ } & \text { K8-09 } \\ \text { Dudder, GB } & \text { K8-29 } \\ \text { Harper, WW } & \text { K5-25 } \\ \text { Hatchell, BK } & \text { K5-22 } \\ \text { Schultz, JF (26) } & \text { K5-25 } \\ \text { Sheen, DM } & \text { K5-25 } \\ \text { Stewart, TL } & \text { K5-25 } \\ \text { Strasburg, JD } & \text { K5-25 } \\ \text { Taubman, MS } & \text { K5-25 } \\ \text { Information Release Office (7) } & \text { K1-06 }\end{array}$

